\title{
Log-periodic route to fractal functions
}

\author{
S. Gluzman ${ }^{1}$ and D. Sornette ${ }^{1,2.3}$ \\ ${ }^{1}$ Institute of Geophysics and Planetary Physics \\ University of California Los Angeles, Los Angeles, CA 90095-1567 \\ ${ }^{2}$ Department of Earth and Space Sciences, UCLA \\ ${ }^{3}$ Laboratoire de Physique de la Matière Condensée \\ CNRS UMR 6622 and Université de Nice-Sophia Antipolis, 06108 Nice Cedex 2, France
}

September 13, 2018

\begin{abstract}
Log-periodic oscillations have been found to decorate the usual power law behavior found to describe the approach to a critical point, when the continuous scale-invariance symmetry is partially broken into a discrete-scale invariance (DSI) symmetry. For Ising or Potts spins with ferromagnetic interactions on hierarchical systems, the relative magnitude of the log-periodic corrections are usually very small, of order $10^{-5}$. In growth processes (DLA), rupture, earthquake and financial crashes, they are found of the order of $10 \%$. We offer a "technical" explanation for this 4-order-of-magnitude difference based on the property of the "regular function" $g(x)$ embodying the effect of the microscopic degrees of freedom summed over in a renormalization group (RG) approach $F(x)=g(x)+$ $\mu^{-1} F(\gamma x)$ of an observable $F$ as a function of a control parameter $x$. For systems for which the RG equation has not been derived, the previous equation can be understood as a Jackson $q$-integral, which is the natural tool for describing discrete scale invariance. We classify the "Weierstrass-type" solutions of the RG into two classes characterized by the amplitudes $A_{n}$ of the power law series expansion. These two classes are separated by a novel "critical" point. Growth processes (DLA), rupture, earthquake and financial crashes thus seem to be characterized by oscillatory or bounded regular microscopic functions that lead to a slow power law decay of $A_{n}$, giving strong log-periodic amplitudes. If in addition, the phases of $A_{n}$ are ergodic and mixing, the observable presents self-affine non-differentiable properties. In contrast, the regular function of statistical physics models with "ferromagnetic"type interactions at equibrium involves unbound logarithms of polynomials of the control variable that lead to a fast exponential decay of $A_{n}$ giving weak log-periodic amplitudes and smoothed observables. These two classes of behavior might be traced back to the existence or abscence of "antiferromagnetic" or "dipolar"-type interactions which, when present, make the Green functions non-monotonous oscillatory and favor spatial modulated patterns.
\end{abstract}




\section{Introduction}

The existence of log-periodic oscillatory corrections to the power laws associated with critical phenomena and, more generally, to observables of systems endowed with the scale-invariance symmetry has been recognized since the 1960s (see [1] for a recent review and references therein). The log-periodic oscillations result from a partial breakdown of the continuous scale-invariance symmetry into a discrete scale-invariance symmetry, as occurs for instance in hierarchical lattices.

However, for one of the most studied class of models exhibiting these oscillations, i.e., Potts model with ferromagnetic interactions on hierarchical lattices, the relative magnitude of the log-periodic corrections are usually very small, of order $10^{-5}$ [2]. In contrast, in growth processes (DLA) [3], 勾, rupture [5], earthquakes [6] and financial crashes [1], 8], they are found of amplitude of the order of $10 \%$.

Here, we propose an explanation for this puzzling observation of an 4-order-of-magnitude difference based on the nature of the microscopic interactions of the systems. Within a renormalization group (RG) approach, an observable at one scale can be related by a functional relation to the same observable at another scale, with the addition of the contribution of the degrees of freedom left-over by the procedure of decimation or of change of scale. This contribution is called the "regular part" of the renormalization group equation of the observable. For systems for which the RG equation has not been derived, the RG equation can be understood without reference to the RG as a Jackson $q$-integral [9], which is the natural tool [10, 11] for describing discrete scale invariance. Here, we do not discuss the mechanisms by which the continuous scale invariance symmetry is broken to give discrete scale invariance but rather present a phenomenological approach based on the functional RG/Jackson $q$-integral equation.

Using the Mellin transform applied to the formal series solution of the renormalization group, we identify two broad classes of systems based on the nature of the decay with order $n$ of the amplitudes $A_{n}$ of the power law series expansion of the observable:

1. systems with quasi-periodic "regular part" and/or with compact support have coefficients $A_{n}$ decaying as a power law $A_{n} \sim n^{-p}$, leading to strong log-periodic oscillatory amplitudes; if in addition, the phases of $A_{n}$ are ergodic and mixing, the observable presents singular properties everywhere, similar to those of 'Weierstrasstype" functions.

2. systems with non-periodic "regular part" with unbound support have $A_{n}$ decaying as an exponential $A_{n} \sim$ $e^{-\kappa n}$ of their order $n$, leading to exceedingly small log-periodic oscillatory amplitudes and regular smooth observables.

We find families of "regular parts" which belong to both classes, with a "critical" transition from the first to the other as a parameter is varied.

A known example of a system of the first class is the $q$-state Potts model with antiferromagnetic interactions [12, 13]. Another example is the statistics of closed-loop self-avoiding walks per site on a family of regular fractals with a discrete scale-invariant geometry such as the Sieirpinsky gasket [14]. A known example of the second class is the $q$-state Potts model with ferromagnetic interactions [2]. Based on this knowledge and on the analysis of this paper which underlines the importance of the oscillatory property of the "regular part" or of its compact support to generate strong log-periodic structure, we conjecture that systems that possess "antiferromagnetic"-type or "dipolar"type interactions that introduce modulated spatial patterns in the system belongs to the first class. This provides a possible explanation for the strong log-periodic oscillatory amplitudes observed empirically in growth processes, rupture, earthquake and financial crashes as we argue in the concluding section.

The next section 2 introduces the renormalization group with a single control parameter, its formal solution with the presence of log-periodic corrections in the presence of discrete scale invariance. Section 3 uses the Mellin transform to resum the formal series solution of the renormalization group into a power law series. Section 4 presents the general classification within the two classes alluded to above in terms of the leading exponential or power law decay of the coefficients of this power law expansion. It examines the conditions under which the observable can develop 
non-differentiable fractal properties similar to Weierstrass-type functions. A family of "regular parts" is introduced which exhibits a critical transition between the two classes. Section 5 presents many more examples of both classes. Section 6 concludes with a qualitative argument explaining why rupture and financial systems should belong to the first class. The table 1 offers a synthesis of the classification in terms of the decay of the coefficients $A_{n}$ of the power series expansion of the observable for various choices of the "regular part" of the renormalization group.

\section{2 "Weierstrass-type functions" from discrete renormalization group equations}

Speaking about a material shape, a mathematical object or function, the symmetry of scale invariance refers to their invariance with respect to changes of scales of observation (see [15, 16] for general introductions). In a nutshell, scale invariance simply means reproducing itself on different time or space scales. Specifically, an observable $f$ which depends on a "control" parameter $x$ is scale invariant under the arbitrary change $x \rightarrow \gamma x$ if there is a number $\mu(\gamma)$ such that

$$
f(x)=\frac{1}{\mu} f(\gamma x) .
$$

Such scale invariance occurs for instance at the critical points $t=t_{c}$ of systems exhibiting a continuous phase transition. The renormalization group theory has been developed to provide an understanding of the emergence of the self-similar property (1) from a systematic scale change and spin decimation procedure [17].

Calling $K$ the coupling (e.g. $K=e^{J / T}$ for a spin model where $J$ is interaction coefficient and $T$ is the temperature) and $R$ the renormalization group map between two successive magnification steps, the free energy $f$ per lattice site, bond, atom or element obeys the self-consistent equation:

$$
f(K)=g(K)+\frac{1}{\mu} f[R(K)],
$$

where $g$ is a regular part which is made of the free energy of the degrees of freedom summed over between two successive renormalizations, $\mu>1$ is the ratio of the number of degrees of freedom between two successive renormalizations. In general, this relationship (2) is an approximation whose validity requires the study of the impact of many-body interactions. When these higher-order interactions can be considered secondary as the scale of description increases (corresponding to so-called "irrelevant" operators), expression (2) becomes asymptotically exact at large scales. For perfectly self-similar problems, for instance for physical systems with nearest-neighbor interactions defined on regular geometrical fractals such as the Cantor set, the Sierpinsky Gasket, etc., or on regular hierarchical lattices, expression (2) is exact at all scales.

It is solved recursively by

$$
f(K)=\sum_{n=0}^{\infty} \frac{1}{\mu^{n}} g\left[R^{(n)}(K)\right],
$$

where $R^{(n)}$ is the $n^{t h}$ iterate of the renormalization transformation. Around fixed points $R\left(K_{c}\right)=K_{c}$, the renormalization group map can be expanded up to first order in $K-K_{c}$ as $R(K)=\gamma\left(K-K_{c}\right)$. Posing $x=K-K_{c}$, we have $R^{(n)}(x)=\gamma^{n} x$ and the solution (3) becomes

$$
f(x)=\sum_{n=0}^{\infty} \frac{1}{\mu^{n}} g\left[\gamma^{n} x\right] .
$$

In principle, (4) is only applicable sufficiently "close" to the critical point $x=0$, that the higher-order terms in the expansion $R(K)=\gamma\left(K-K_{c}\right)$ can be neglected. The effect of nonlinear corrections terms for $R(K)$ have been considered in [12, 2].

The form (3) or (4) has not been derived rigorously for growth, rupture and other out-of-equilibrium processes alluded to above, even if there are various attempts to develop approximate RG descriptions on specific models of 
these processes. It may thus seem a little premature to use this discrete renormalization group description for these systems. Actually, expression (4) can be obtained without any reference to a renormalization group approach: as soon as the system exhibits a discrete scale invariance, the natural tool is provided by $q$-derivatives [11] from which it is seen that expression (4) is nothing but a Jackson $q$-integral [9] of the function $g(x)$, which constitutes the natural generalization of regular integrals for discretely self-similar systems [11]. The way the Jackson $q$-integral is related to the free energy of a spin system on a hierarchical lattive was explained in [18].

In the mathematical literature, the function ( 4 ) is called a Weierstrass-type function, to refer to the introduction by K. Weierstrass of the function [19]

$$
f_{W}=\sum_{n=0}^{\infty} b^{n} \cos \left[a^{n} \pi x\right]
$$

corresponding to the special case $\mu=1 / b, \gamma=a$ and $g(x)=\cos [\pi x]$. To the surprise of mathematicians of the 19th century, Weierstrass showed that the function (5) is continuous but differentiable nowhere, provided $0<b<1, a>1$ and $a b>1+\frac{2}{3} \pi$. Note that, in the context of the renormalization group of critical phenomena, the condition $a=\gamma>1$ implies that the fixed point $K_{c}$ is unstable. Hardy was able to improve later on the last bound and obtain that the Weierstrass function (5) is non-differentiable everywhere as soon as $a b>1$ [20]. In addition, Hardy showed that it satisfies the following Lipschitz condition (corresponding to self-affine scaling) for $a b>1$, which is much more than just the statement of non-differentiability:

$$
f_{W}(x+h)-f_{W}(x) \sim|h|^{m}, \text { for all } x \text { where } m=\ln [1 / b] / \ln a .
$$

Note that for $a b>1, m<1$, expression (6) shows that $f_{W}(x+h)-f_{W}(x) \gg|h|$ for $h \rightarrow 0$. As a consequence, the ratio $\left[f_{W}(x+h)-f_{W}(x)\right] / h$ has no limit which recovers the property of non-differentiability. Continuity is obvious from the fact that $f_{W}(x+h)-f_{W}(x) \rightarrow 0$ as $h \rightarrow 0$ since $m>0$. For the border case $a=b$ discovered by Cellerier before 1850, $f_{W}$ is not non-differentiable in a strict sense since it possesses infinite differential coefficients at an everywhere dense set of points [21]. Richardson is credited with the first mention of the potential usefulness for the description of nature of the continuous everwhere non-differentiable Weierstrass function [22]. Shlesinger and co-workers [23] have previously noticed and studied the correspondence between (4) and Weierstrass function.

If one is interested in the non-regular (or non-analytic) behavior only close to the critical point $x=0$, the regular part can be dropped and the analysis of (11) is sufficient. It is then easy to show that the most general solution of (11) is (see [1] and references therein)

$$
f(x)=x^{m} P\left(\frac{\ln x}{\ln \gamma}\right)
$$

where

$$
m=\frac{\ln \mu}{\ln \gamma},
$$

and $P(y)$ is an arbitrary periodic function of its argument $y$ of period 1. Its specification is actually determined by the regular part $g(x)$ of the renormalization group equation, as shown for instance in the explicit solution (4). The scaling law $f(x) \sim x^{m}$ implied by (7) is a special case of (6) obtained by putting $x=0$ and replacing $h$ by $x$ in (6).

The Laplace transform $f_{L}(\beta)$ of $f(x)$ defined by (円) also obeys a renormalization equation of the type (2). Denoting $g_{L}(\beta)$ the Laplace transform of the regular part $g(x)$, we have

$$
f_{L}(\beta)=\sum_{n=0}^{\infty} \frac{1}{(\mu \gamma)^{n}} g_{L}\left[\beta / \gamma^{n}\right],
$$

and

$$
f_{L}(\beta)=g_{L}(\beta)+\frac{1}{\mu \gamma} f_{L}\left(\frac{\beta}{\gamma}\right)
$$


The general solution of (10) takes the same form as (7):

$$
f_{L}(\beta)=\frac{1}{\beta^{1+m}} P_{L}\left(\frac{\ln \beta}{\ln \gamma}\right)
$$

where $P_{L}(y)$ is an arbitrary periodic function of its argument $y$ of period 1.

\section{Reconstruction of "Weierstrass-type functions" from power series expansions}

Following [2, 24], we use the Mellin transform to obtain a power law series representation of the Weierstrass-type function (\$). The Mellin transform is defined as

$$
\hat{f}(s) \equiv \int_{0}^{\infty} x^{s-1} f(x) d x .
$$

The Mellin transform (12) provides a reconstruction of the infinite sum of the Weierstrass-type function ( 4 ) as a sum of power law contributions, $A_{n} x^{-s_{n}}$, with "universal" complex exponents $s_{n}$ determined only by properties of the hierarchical construction and not by the function $g(x)$, with amplitudes $A_{n}$ controlled by the form of the regular part $g(x)$. These "non-universal" amplitudes in turn control the shape of the function $f(x)$, its differentiability or non-differentiability as well as its self-affine (fractal) properties, as we shall describe in the sequel.

The Mellin transform of (円) reads

$$
\widehat{f}(s)=\frac{\mu \gamma^{s}}{\mu \gamma^{s}-1} \widehat{g}(s),
$$

where $\widehat{g}(s)$ is the Mellin transform of $g(x)$. The inverse Mellin transformation of $\widehat{f}(s)$,

$$
f(x)=\frac{1}{2 \pi i} \int_{c-i \infty}^{c+i \infty} \widehat{f}(s) x^{-s} d s
$$

allows us to reconstruct $f(x)$ as a new expansion in singular as well as regular powers of $x$ in order to unravel its selfsimilar properties. Indeed, the usefulness of the Mellin transform is that power law behaviors spring out immediately from the poles of $\widehat{f}(s)$, using Cauchy's theorem.

In inverting the Mellin transform, we have two types of poles. The poles of the Mellin transform $\hat{g}$ of the analytical function $g(x)$ occur in general at integer values and contribute only to the regular part $f_{r}(x)$ of $f(x)$, as expected since

$g(x)$ is a regular contribution. The poles of the first term $\frac{\mu \gamma^{s}}{\mu \gamma^{s}-1}$ in the r.h.s. of (13) stem from the infinite sum over successive embeddings of scales and occur at $s=s_{n}$ where

$$
s_{n}=-m+i \frac{2 \pi}{\ln \gamma} n
$$

and $m$ is given by (8). Their amplitude $A_{n}$ is obtained by applying Cauchy's theorem and is given by the residues

$$
\lim _{s \rightarrow s_{n}} \frac{s-s_{n}}{\mu \gamma^{s}-1} \widehat{g}(s)=\frac{\exp (-2 \pi n i)}{\ln \gamma} \widehat{g}(s)=\frac{\widehat{g}(s)}{\ln \gamma} .
$$

The resulting expression for $f(x)$ is

$$
f(x)=f_{s}(x)+f_{r}(x),
$$

where the singular part $f_{s}(x)$ is given by

$$
f_{s}(x)=\sum_{n=0}^{\infty} A_{n} x^{-s_{n}}
$$


and

$$
A_{n}=\frac{\widehat{g}\left(s_{n}\right)}{\ln \gamma} .
$$

This approach is similar to the one developed in [25] for "fractal strings" $\eta$ (for instance, the complementary of the triadic Cantor set is a special fractal string). Their fractal properties are fully characterized by the introduction of the "geometric zeta function" $\zeta_{\eta}(s)$, which can be shown to be nothing but the Mellin transform of the measure defined on the fractal string (see [25] page 73). In particular, the poles of $\zeta_{\eta}(s)$ give the complex fractal dimensions of the fractal strings, similarly to the role played here by the complex exponents $s_{n}$ defined by (15).

The regular part $f_{r}(x)$ of $f(x)$ defined in (17) is generated by the poles of $\widehat{g}(s)$ if any, located at $s=-n$, $n=0,1 \ldots$ The residues of these poles give the coefficients $B_{n}$ of the expansion of the regular part as follows:

$$
f_{r}(x)=\sum_{n=0}^{\infty} B_{n} x^{n}
$$

\section{Classification of "Weierstrass-type functions"}

\subsection{Classification}

The representation (18) offers a classification of "Weierstrass-type functions" as follows. We will work in the class of $g(x)$ (not covering of course all possible types of behavior of $A(n)$ ) where the coefficients $A_{n}$ can be expressed as the product of an exponential decay by a power prefactor and a phase

$$
A_{n}=\frac{1}{\ln \gamma} \frac{1}{n^{p}} e^{-\kappa n} e^{i \psi_{n}}, \quad \text { for large } n,
$$

where $p, \kappa \geq 0$ and $\psi_{n}$ are determined by the form of $g(x)$ and the values of $\mu$ and $\gamma$. This class is broad enough to include many physically interesting shapes of $g(x)$ as will be illustrated at length below.

\subsubsection{Justification of the classification}

The parameterization (21) can be seen to result from very general theorems on the Mellin transform [26, 27]. Let us assume that the function $g(x)$ defined for $x>0$ is continuous and satisfies the following conditions

$$
\mid g\left(x | \leq c _ { 1 } x ^ { \alpha } , \quad 0 < x \leq 1 ; \quad | g \left(x \mid \leq c_{2} x^{\beta}, \quad 1 \leq x<\infty,\right.\right.
$$

where $\alpha>\beta$. Then, its Mellin transform is a regular (differentiable) function inside the strip $-\alpha<\operatorname{Re}(s)<-\beta$, One should also bear in mind that $\operatorname{Re}(s)<0$ because of the constraints imposed by the very formulation of the problem. All functions that we shall consider below as examples belong to the class of continuous functions satisfying slightly more restricted conditions [26] such as (22) with $\alpha>0$ and $\beta=0$. As a consequence, their Mellin transform is regular for $-\alpha<\operatorname{Re}(s)<0$. For instance, $g(x)=\cos (x)-1$ corresponds to $\alpha=1$ and $\beta=0$. The same conditions apply to $\log (1+x)$ and $\exp (-x)-1$. For the stretched exponential function $\exp \left(-x^{h}\right)-1$ with $\left.h>0\right)$, we have $\alpha=h$ and again $\beta=0$.

We are interested, in $g \widehat{\left(s_{n}\right)}=\widehat{g}(-m+i n \omega)$, particularly as $n$ goes to infinity. The general condition which is usually imposed on this quantity in order to ensure the existence of its inverse Mellin transform is [27]

$$
\widehat{g}(-m+i n \omega) \rightarrow 0, \quad \text { as } n \rightarrow+\infty .
$$

Again, $A_{n}$ must be designed in such a way that it satisfies this condition automatically.

Let us consider some simple but vivid examples, intended to illustrate how a power-law and exponential decay of $A_{n}$ as a function of $n$ emerges from simple functions satisfying the conditions stated above. We also note that, when 
$g(t)$ possesses discontinuities of the first kind, it still yields the dependence (21) of $A_{n}$ as a function of $n$. Maybe the simplest function leading to $A_{n}$ with a power-law decay is

$$
g(x)=0, \quad 0<x<1 \text { and } g(x)=-1, \quad 1<x<\infty,
$$

which leads to $\widehat{g(s)}=\frac{1}{s}$, which is regular within the strip $\infty<\operatorname{Re}(s)<0$. The corresponding $A_{n}$ decays in amplitude as $n^{-1}$ for large $n$. Different strip geometries lead to the same power-law decay of $A_{n}$, for instance

$$
g(x)=x^{a}, \quad 0<x<1 \quad \text { and } \quad g(x)=0, \quad 1<x<\infty,
$$

with Mellin transform $\widehat{g(s)}=(s+a)^{-1}$ with $-a<\operatorname{Re}(s)<0$. Let us also consider

$$
g(x)=0, \quad 0<x<1 \quad \text { and } g(x)=-x^{a}, \quad 1<x<\infty,
$$

which leads to a similar Mellin transform $\widehat{g(s)}=(s+a)^{-1}$ but a different strip geometry $\operatorname{Re}(s)<-a$. The slightly more complicated example of a continuous function composed of power laws

$$
g(x)=(b-a)^{-1} x^{a}, \quad 0<x<1 \quad \text { and } \quad g(x)=(b-a)^{-1} x^{b}, \quad 1<x<b,
$$

leads to $\widehat{g(s)}=(s+a)^{-1}(s+b)^{-1}$ with $-a<\operatorname{Re}(s)<-b$ and the amplitude of $A_{n}$ decaying as $n^{-2}$. The analysis of these examples and of their Mellin transforms at $s=s_{n}$ demonstrate that particulars of the strip geometry in the variable $s$ are not important when one is concerned with the large $n$ asymptotic behavior of $\widehat{g}\left(s_{n}\right)$. The asymptotic power decay of $A_{n}$ as a function of $n$ can be dominated by an exponential decay, as we shall see in more details below. For instance, the continuous function formed by compounded power-laws $g(x)=\frac{1}{\pi} x(1+x)^{-1}$ leads to $\widehat{g(s)}=-\csc (\pi s), \quad-1<\operatorname{Re}(s)<0$, yielding $A_{n}$ decaying as $\exp (-n)$ as $n \rightarrow+\infty$.

Violations of the parameterization (21) regarding $A_{n}$ occur when the conditions of the theorem [26] are changed, e.g. when the argument $x$ is replaced by, say, $\ln (1 / x)$ or when singularities are introduced to the function $g(x)$. This can be seen from equation (19) which shows that $A_{n}$ is proportional to the Mellin transform of $g(t)$ expressed at $s=s_{m}=-m+i n \omega$ where

$$
\omega=\frac{2 \pi}{\ln \gamma} .
$$

Posing $u=\ln x$, the Mellin transform becomes a Fourier transform

$$
A_{n}=\frac{1}{\ln \gamma} \int_{-\infty}^{+\infty} d u G(u) e^{i \omega n u}
$$

where

$$
G(u) \equiv e^{u(1-m)} g\left(e^{u}\right) .
$$

It is clear that, by a suitable choise of $g(x)$, any dependence of $A_{n}$ can be obtained. For instance, for

$$
G(u)=u^{-3 / 2} e^{-a / u},
$$

we obtain $A_{n} \sim e^{-\sqrt{2 a n}} \cos [\sqrt{2 a n}]$, which exhibits an oscillatory stretched-exponential decay intermediate between the exponential $(\kappa>0)$ and pure power law decay $(\kappa=0)$ of (21). However, the choice (31) corresponds to a rather special choice for

$$
g(x)=\frac{(\ln x)^{-3 / 2} e^{-a / \ln x}}{t^{1-m}} .
$$

In this case, $g(x) \rightarrow+\infty$ for $x \rightarrow 0$, and this case is outside the domain of validity (22) of the theorem [26, 27]. Consider also the following example

$$
g(x)=\pi^{-1 / 2} \exp \left(-\frac{\ln (1 / x)^{2}}{4}\right)
$$


leading to $\widehat{g(s)}=\exp \left(s^{2}\right)$, valid for arbitrary $s$, which leads to $A_{n}$ with amplitude decaying as $\exp \left(-n^{2}\right)$ as $n \rightarrow \infty$. This example is also characterized by a pathological behavior for $x \rightarrow+\infty$ of $g(x)$ which diverges faster than any power law. Another pathological example is

$$
g(x)=1 / 2 \pi^{-1 / 2} \cos \left((1 / 4) \ln (1 / x)^{2}-\pi / 4\right),
$$

leading to $\widehat{g(s)}=\cos s^{2}$, valid for arbitrary $s$, which yields $A_{n}$ with an amplitude growing as $\exp (n)$ as $n \rightarrow+\infty$. This violates the condition on the Mellin transform given in [27].

The existence of discontinuities of $g(x)$, as one might expect from the theorem [26, 27], also violates the parameterization (21) of $A_{n}$. Consider $g(x)=-\frac{1}{\pi} x^{1 / 2}(1-x)^{-1}$ with an integrable singularity, which gives $\widehat{g(s)}=$ $\tan (\pi s), \quad-1 / 2<R e(s)<0$ and $A_{n}$ with an amplitude bounded from below by a constant as $n \rightarrow+\infty$. This absence of decay allows us to reject this type of function, since a decay of $A_{n}$ is required by [27], In constrast, a logarithmic singularity, as for instance in $g(x)=\frac{1}{\pi} \log \left|\frac{1+x}{1-t}\right|$, is allowed. In this case, this gives $\widehat{g(s)}=$ $s^{-1} \tanh (\pi s),-1<R e(s)<0$ and the amplitude of $g \widehat{\left(s_{n}\right)}$ exhibits periodic modulations as $n \rightarrow+\infty$ as

$n^{-1}\left(\sinh ^{2}\left(\frac{1}{2} \pi m\right)+\cos ^{2}\left(\frac{1}{2} \pi \omega n\right)\right)^{-1}(\sin (\pi \omega n)+i \sinh (\pi m))$. Another example with the logarithmic function (93) discussed below gives a power law decay with a logarithmic correction as shown by (95) due to the presence of the singularity.

In conclusion, as long as the conditions of theorem [26] on Mellin transforms hold, the dependence of $A_{n}$ as $n \rightarrow+\infty$ given by (21) will hold as well. Violations of the theorem due to a change of variable or to the presence of simple poles lead either to a faster decay or to a non-decaying $A_{n}$. Allowing for logarithmic singularities within $g(x)$ brings in logarithmic or oscillatory corrections to $A_{n}$ as a function of $n$.

\subsubsection{Beyond the linear approxiation of the renormalization group map}

The asymptotic expansion (21) uses the linear approximation $R^{(n)}(x)=\gamma^{n} x$ that allows us to transform the general solution (3) into the "Weierstrass-type function" (4). As we said, (4) is only applicable sufficiently "close" to the critical point $x=0$, such that the higher-order terms in the expansion $R(x)=\gamma x$ can be neglected. The linear approximation of $R^{(n)}(x)=\gamma^{n} x$ is bound however to become incorrect as $n$ becomes very large, i.e., in the region determining the singular behavior. As discussed in [2, 24], the crucial property missed by the linear approximation is that $f(x)$ is analytic only in a sector $|\arg x|<\theta$ while we treated it as analytic in the cut plane $|\arg x|<\pi$. This implies that the exponential contribution $e^{-\kappa n}$ of the true asymptotic decay of the amplitudes of successive log-periodic harmonics is slower than found from the linear approximation, and goes as $e^{-\kappa \theta n}$. The angle $\theta$ depends specifically on the flow map $R(x)$ of the discrete renormalization group [2] and is generally of order 1. Our classification in two sets $\kappa=0$ and $\kappa \neq 0$ is not modified by this subtlety. Here, we shall consider only the "Weierstrass-type functions" (4) and will revisit the impact of nonlinear terms of the renormalization group map in a future communication.

\subsection{3 $\kappa>0: C^{\infty}$-differentiability}

The general solution (7) remains true for any choice of the regular part $g(x)$ with the exponent $m$ given by (8). This implies that there will always be an order of differentiation sufficiently large such that its becomes infinite at $x=0$ [12]. This is the crux of the argument on the existence of the singularity at $x=0$. Here, we investigate the differentiability of $f(x)$ for non-zero values of $x$, i.e., away from the unstable critical point $x \rightarrow 0$. Expression (18) with (15) provides a direct way for understanding the origin of the singular behavior at $x \rightarrow 0$, as $x^{m}$ is in factor of an infinite sum of oscillatory terms with log-periodic oscillations condensing geometrically as $x \rightarrow 0$.

When $\kappa>0$, the modulus of $A_{n}$ decay exponentially fast to zero. Hence, $f(x)$ is differentiable at all orders. This can be seen from the fact that

$$
\frac{d^{\ell} f_{s}(x)}{d x^{\ell}}=\sum_{n=0}^{\infty}\left(-s_{n}\right)\left(-s_{n}-1\right) \ldots\left(-s_{n}-\ell+1\right) A_{n} x^{-s_{n}-\ell}
$$


is absolutely convergent for any order $\ell$ of differentiation. Taking into account that $\left|x^{-s_{n}-\ell}\right|=x^{m-\ell}$ is independent of $n$ and can be factorized, the $n$th term in the sum has an amplitude bounded by a constant times $n^{\ell} \exp [-q n]$ since $\left(-s_{n}\right)\left(-s_{n}-1\right) \ldots\left(-s_{n}-\ell+1\right)$ is bounded from above by a constant times $n^{\ell}$. The sum is thus controlled by the exponentially fast decaying coefficients $A_{n}$ and converges to well-defined values for any $\ell$. As a consequence of the exponential decay of the coefficients $A_{n}$, the log-periodic oscillations are extremely small.

Another obvious way to ensure differentiability even when $\kappa=0$ (see next section) is to truncate the number $n$ of powers $x^{-s_{n}}$ in the sum (18) to a finite value:

$$
f_{s}^{(N)}(x)=\sum_{n=0}^{N} A_{n} x^{-s_{n}},
$$

An example with $N=1,2,3$ is shown in figure 1 for the Weierstrass function $\left(\alpha=\pi / 2\right.$ and $\left.p=m+\frac{1}{2}\right)$. For $N=1$, the real part $f_{s}^{(1)}(x)$ is given by

$$
f_{s}^{(1)}(x)=a_{0}\left[1+\frac{A_{n=0}}{a_{0}} x^{m}+\frac{\left|A_{n=1}\right|}{a_{0}} x^{m} \cos (\omega \ln (x)+\varphi)\right], \quad a_{0}=\frac{\mu}{\mu-1},
$$

where $\omega$ is given by $(28)$ and

$$
\varphi=\arctan \left(\frac{\operatorname{Im}\left(A_{n=1}\right)}{\operatorname{Re}\left(A_{n=1}\right)}\right)+k \pi, \quad k=0, \pm 1 \ldots
$$

This expression (37) is based on the singular part (18) of the Mellin decomposition of the DSI equation (4). It applies not only to the Weierstrass function but to any function of the form (đ). Keeping only the first two terms recovers exactly the log-periodic formula introduced in the study of precursors of material failure [28, 29, 5], of earthquakes precursors [6, 30, 31] and of precursors of financial crashes [7, 32].

\subsubsection{Critical behavior and non-differentiability}

Expression (18) with (15) and (21) shows that $f_{s}(x)$ has the same differentiability properties as

$$
\sum_{n=1}^{+\infty} \frac{1}{n^{p}} e^{i \psi_{n}} x^{m-i 2 \pi n / \ln \gamma} .
$$

Changing variable $x \rightarrow y=\ln x / \ln \gamma$, this reads

$$
e^{y \ln \mu} \sum_{n=1}^{+\infty} \frac{1}{n^{p}} e^{i\left(-2 \pi n y+\psi_{n}\right)} .
$$

With respect to the differentiability property, it is sufficient to study the real part of the infinite sum which reads

$$
K_{p,\left\{\psi_{n}\right\}}(y)=\sum_{n=1}^{+\infty} \frac{\cos \left[2 \pi n y+\psi_{n}\right]}{n^{p}} .
$$

This expression allows us to recover some important results in the case where the phases $\psi_{n}$ are sufficiently random so that the numerators $\cos \left[2 \pi n y+\psi_{n}\right]$ take random uncorrelated signs with zero mean. Then, the sum $K_{p,\left\{\psi_{n}\right\}}(y)$ truncated at $n=T$ has the same convergence properties for $T \rightarrow \infty$ as

$$
X(T)=\int_{1}^{T} \frac{d W_{t}}{t^{p}}
$$


where $d W_{t}$ is the increment of the continuous white noise Brownian motion of zero mean and correlation function $\left\langle d W_{t} d W_{t^{\prime}}\right\rangle=\delta(t-t) d t$ where $\delta$ is the Dirac function. We get $\langle X(T)\rangle=0$ and its variance is

$$
\left\langle[X(T)]^{2}\right\rangle=\int_{1}^{T} \int_{1}^{T}\left\langle d W_{t} d W_{t^{\prime}}\right\rangle t^{-p} t^{\prime-p}=\int_{1}^{T} \frac{d t}{t^{2 p}},
$$

which is finite for $T \rightarrow+\infty$ if $p>1 / 2$. This entails the convergence for $p>1 / 2$ of the infinite series (41) for most phases $\psi_{n}$ which are sufficiently ergodic and mixing. We thus expect that $K_{p,\left\{\psi_{n}\right\}}(y)$ and as a consequence $f_{s}(x)$ are continuous functions for $p>1 / 2$. We can proceed similarly for studying their $\ell$ 's derivative. With respect to the convergence property, taking the $\ell$ 's derivative has the effect of changing $p$ into $p-\ell$ in (42). We thus expect $K_{p,\left\{\psi_{n}\right\}}(y)$ and as a consequence $f_{s}(x)$ to be differentiable of order $\ell$ for $p>\ell+1 / 2$.

We conjecture the following.

Conjecture on conditions for nondifferentiability from the singular power law expansion of "Weierstrasstype functions": provided that (1) $\kappa=0$ and (2) the phases $\psi_{n}$ are ergodic and, using a generalization of Hardy's condition $a b>1$ for the Weierstrass function, the smallest order $\ell_{\min }$ of differentiation of $f_{s}(x)$ (defined by $(18)$ with exponents $s_{n}$ given by (15) with (8)) which does not exist is such that

$$
\frac{1}{2}<p-\ell_{\min }<\frac{3}{2}
$$

i.e.,

$$
\ell_{\min }=\operatorname{Int}\left[p-\frac{3}{2}\right]
$$

is the integer part of $p-\frac{3}{2}$. In particular, with ergodic phases $\psi_{n}$ of zero mean, the function $f_{s}(x)$ is nondifferentiable for $p<3 / 2$.

It follows from Lebesgue's theorem on continuous functions of bounded variations that a non-differentiable function is not a function of bounded variation. Therefore, a non-differentiable function is everywhere oscillating and the length of arc between any two points on the curve is infinite [21]. This explains the observation below that the regular part $g(x)$ must contain oscillations or must exhibit a compact support (so that it has a discrete Fourier series) in order for $f(x)$ to be non-differentiable or for some of its derivatives to be non-differentiable. Actually, Weierstrass-type functions (4) are believed to have the same Hausdorff dimension $2-m$ as the Weierstrass function (5) for arbitrary regular part $g(x)$, as long as it is a bounded almost periodic Lipschitz function of order $\beta>m$ [34]. The examples organized below in two classes illustrate and make precise this condition on $g(x)$. We indeed find that non-differentiability occurs at a finite order of differentiation only for functions $g(x)$ which are periodic or with compact support.

It appears however that there is not yet a general understanding whether there exists a necessary and sufficient condition for the differentiability of a function on an interval. It is well-known that continuity is necessary for differentiability but is not sufficient as shown by the Weierstrass function and other examples above. The restriction of bounded variations has also proved insufficient: although a continuous function must possess a differential coefficient almost everywhere, yet there are examples of such functions which do not possess differential coefficients at unenumerable everywhere dense sets of points [21].

\subsection{General condition for $\kappa=0$}

Let us consider a regular function $g(x)$ which is either periodic with period $X$ or with compact support over the interval $[0, X]$ and zero outside. It can then be expanded as a Fourier series

$$
g(x)=\frac{a_{0}}{2}+\sum_{k=1}^{+\infty}\left[a_{k} \cos (2 \pi k x / X)+b_{k} \sin (2 \pi k x / X)\right],
$$


where $a_{0}, a_{1}, b_{1}, \ldots, a_{k}, b_{k}, \ldots$ are arbitrary real numbers.

The behavior of the coefficients $A_{n}$ is controlled by the Mellin transform $\widehat{g}\left(s_{n}\right)$ of $g(x)$ as shown by (19). For $g(x)$ periodic with zero mean, $a_{0}=0$ and

$$
\widehat{g}\left(s_{n}\right)=\left(\frac{X}{2 \pi}\right)^{s_{n}}\left[\widehat{\cos }\left(s_{n}\right) \sum_{k=1}^{+\infty} \frac{a_{k}}{k^{s_{n}}}+\widehat{\sin }\left(s_{n}\right) \sum_{k=1}^{+\infty} \frac{b_{k}}{k^{s_{n}}}\right],
$$

where $\widehat{\cos }(s)$ and $\widehat{\sin }(s)$ are the Mellin transform of $\cos x$ and $\sin x$. Now, a general theorem on the Fourier series of periodic functions tells us that, if $g(x)$ has continuous derivatives up to order $r-1$ included and if the derivative of order $k$ obeys the Dirichlet conditions, then the coefficients $a_{k}$ and $b_{k}$ decay for large $k$ as $1 / k^{r+1}$, i.e., there is finite $M^{\prime}>M>0$ such that $M^{\prime} / k^{r+1}>\left|a_{k}\right|>M / k^{r+1}$ and $M^{\prime} / k^{r+1}>\left|b_{k}\right|>M / k^{r+1}$. If $g(x)$ is discontinuous at a discrete set of points, this corresponds to taking $r=0$ in the previous formula. The Dirichlet conditions are: (i) $g(x)$ is continuous or possess only a finite number of discontinuities; (ii) each point of discontinuity $x_{d}$ is a discontinuity of the first kind, i.e., it is such that the limits to the left $g\left(x \rightarrow x_{d}^{-}\right)$and to the right $g\left(x \rightarrow x_{d}^{+}\right)$are finite; (iii) the interval $[0, X]$ can be divided into a finite set of subintervals on each of which $g(x)$ is monotonous.

We can thus write

$$
2 M\left(\frac{X}{2 \pi}\right)^{s_{n}} \widehat{\sin }\left(s_{n}\right) \sum_{k=1}^{+\infty} \frac{k^{-i n \omega}}{k^{r+1-m}}<\widehat{g}\left(s_{n}\right)<2 M^{\prime}\left(\frac{X}{2 \pi}\right)^{s_{n}} \widehat{\sin }\left(s_{n}\right) \sum_{k=1}^{+\infty} \frac{k^{-i n \omega}}{k^{r+1-m}} .
$$

The sum

$$
\sum_{k=1}^{+\infty} k^{-(r+1-m+i n \omega)}
$$

is nothing else but the celebrated zeta-function $\zeta(y)$ of Riemann [35, 36], with the correspondence $y=\sigma+i t, \sigma=$ $r+1-m, t=n \omega$. It is known [35, 36] that $|\zeta(\sigma+i t)| \leq C_{\sigma}(|t|+1)^{1 / 2-\sigma}$ for $\sigma<0$, where $C_{\sigma}$ decreases like $(2 \pi e)^{\sigma-1 / 2}$ for $\sigma \rightarrow-\infty$, and it does not satisfy a better estimate in this half-plane. For $0 \leq \sigma \leq 1$ (corresponding to $0 \leq m \leq 1$ and $\left.p=0, \zeta(\sigma+i t) \mid \leq K t^{(1-\sigma) / 2} \ln (t)\right)$ uniformly for some constant $K$. However, we need the behavior of $\zeta(\sigma+i t)$ for $\sigma=r+1-m>0$. It is obtained by using the relation $\zeta(s)=2^{s} \pi^{s-1} \sin \frac{\pi s}{2} \Gamma(1-s) \zeta(1-s)$, which which can be separated into two parts that can be evaluated. Namely, $\sin \left(\frac{\pi}{2} s\right) \Gamma(1-s)$, re-casted in the variable $z=1-s$, takes the familiar form, $\cos \left(\frac{\pi}{2} z\right) \Gamma(z)$, which behaves for large $n$ as $n^{-r+m-1 / 2}$. The other term $\zeta(1-s)=\zeta(z)$ can be evaluated using the expression presented above for the zeta-function of an argument with negative real part (in our case for negative $1-\sigma$ and large $n$ ), $\zeta(z) \leq C(|n|+1)^{1 / 2-(1-\sigma)}=C(|n|+1)^{1 / 2+r-m}$. Therefore, the product of these two terms is of the order of $\mathrm{C}$ and the whole sum decay is slower than exponential.

This shows that the sum (49) is of order $O\left(1 / n^{r+(1 / 2)-m}\right)$ and thus $\widehat{g}\left(s_{n}\right)$ is asymptotically a negative power of $n$ for large $n$. This demonstrates that any periodic continuous function $g(x)$ leads to a power law decay for $A_{n}$ as a function of $n$. The continuity of $g(x)$ implies that $r \geq 1$, ensuring that $r+(1 / 2)-m>0$ for $m<1$.

The same approach can be used for $g(x)$ not periodic but defined on a compact support $[0, X]$. The discrete Fourier series expansion (46) still holds for $x \in[0, X]$ while $g(x)=0$ for $x$ outside. A similar expression to (47) then holds in which $a_{0} \neq 0$ in general and in which the Mellin transforms $\widehat{\cos }(s)$ and $\widehat{\sin }(s)$ are defined over the interval $[0, X]$.

\subsection{Bifurcation from wild to smooth "Weierstrass-type functions": an example using damped oscil- lators for the regular part of the renormalization group equation}

As a first example, let us consider the regular part $g(x)$ of the renormalization group equation defined as

$$
g(x)=e^{-\cos (\alpha) x} \cos (x \sin (\alpha)), \quad \text { with } \alpha \in\left[0, \frac{\pi}{2}\right]
$$


The parameter $\alpha$ quantifies the relative strength of the oscillatory structure of $g(x)$ versus its "damping": for $\alpha=\pi / 2$, (4) with (50) recovers the initial function (5) introduced by Weierstrass with $b=1 / \mu, a=\gamma$ and $\cos (\pi x)$ replaced by $\cos (x)$; for $\alpha=0, g(x)=\exp [-x]$ has no oscillation anymore and corresponds to a pure exponential relaxation considered in [33].

Plugging (50) in (4) gives

$$
f(x)=\sum_{n=0}^{\infty} \frac{1}{\gamma^{(2-D) n}} e^{-\cos (\alpha) \gamma^{n} x} \cos \left(\gamma^{n} x \sin (\alpha)\right),
$$

where

$$
D=2-m=2-\frac{\ln \mu}{\ln \gamma} .
$$

The exponent $D$ turns out to be equal to the fractal dimension of the the graph of the Weierstrass function obtained for $\alpha=\pi / 2$. Recall that the fractal dimension quantifies the self-similarity properties of scale invariant geometrical objects. Note that $1<D<2$ as $1<\mu<\gamma$ which is the condition of non-differentiability found by Hardy [20] for the Weierstrass function. The graph of the Weierstrass function is thus more than a line but less than a plane. For $\alpha<\pi / 2, f(x)$ is smooth and non-fractal $(D=1)$ and its graph has the complexity of the line. Actually, there are several fractal dimensions. It is known that the box counting (capacity, entropic, fractal, Minkowski) dimension and the packing dimensions of the Weierstrass function are all equal to $D$ [37] given by (52) for $\alpha=\pi / 2$. It is conjectured but not proved that the Hausdorff fractal dimension of the graph of the Weierstrass function obtained for $\alpha=\pi / 2$ is also equal to $D$ given by (52). It is known that the Hausdorff dimension of the graph of $f(x)$ does not exceed $D$ but there is no satisfactory condition to estimate its lower bound [34.

Figure 2 shows the function (51) for $\alpha=\pi / 2=1.5708$ (pure Weierstrass function: panel a), $\alpha=0.993 \pi / 2=$ 1.56 (panel b), $\alpha=0.9 \pi / 2=1.414$ (panel c) and and $\alpha=0$ (panel d).

The Mellin transform of $g(x)$ defined by (50) for $-1<\operatorname{Re}[s]=-m<0$ (which is the interval of interest, as seen from (15)) is [38]

$$
\widehat{g}(s)=\Gamma(s) \cos (\alpha s)-\frac{1}{s},
$$

where $\Gamma(s)$ is the Gamma function reducing to $\Gamma(s)=(s-1)$ ! for integer arguments $s$. The additional term $-1 / s$ disappears for $0<\operatorname{Re}[s]$. For values of the exponent $m$ larger than 1, i.e., $\operatorname{Re}[s]<-1$, additional correction terms should be added to (53) [38]. These additional terms only contribute to the power law dependence of the amplitudes $A_{n}$ and not to the exponential. This problem is absent when the cosine in the definition of $g(x)$ is replaced by the sine function.

As we shall discuss below, the modification of $g(x)$ into the modified function

$$
g_{M}(x)=e^{-\cos (\alpha) x} \cos (x \sin (\alpha))-1
$$

gives $\widehat{g}(s)=\Gamma(s) \cos (\alpha s)$ without the correction $-1 / s$ for $-1<\operatorname{Re}[s]=-m<0$ and leads to the so-called Mandelbrot-Weierstrass function. Similar "counter-term" should be introduced for stretched exponential and in similar cases. They do not bring any extra contributions to the Mellin transform.

The regular part $f_{r}(x)$ defined by (20) of $f(x)$ defined in (17) corresponding to $g(x)$ defined by (50) is generated by the poles of $\Gamma(s)$, located at $s=-n, n=0,1 \ldots$, since $\Gamma(s)$ is analytic on the whole complex plane excluding these simple poles [39]. Using the expression $\operatorname{Re} s_{s=-n} \Gamma(s)=\frac{(-1)^{n}}{n !}$, we obtain its explicit form (20) with

$$
B(n)=\frac{(-1)^{n}}{n !} \frac{\mu}{\mu-\gamma^{n}} \cos (\alpha n) .
$$

Note the particularly simple expression of the first term $B_{0}=\frac{\mu}{\mu-1}$. For $|x| \ll 1$, this constant term provides the only non-negligible contribution of the regular part $f_{r}(x)$ to $f(x)$, whose behavior is completely controlled by the sum $f_{s}(x)$ of singular power laws. 
The amplitudes $A_{n}$ defined by (19) corresponding to $g(x)$ defined by (50) are

$$
A_{n}(\alpha)=\frac{\Gamma\left(s_{n}\right) \cos \left(\alpha s_{n}\right)}{\ln \gamma}
$$

The singular part $f_{s}(x)$, which is defined by (18) where the exponents $s_{n}$ are given by (15), satisfies the exact scaleinvariance equation (1).

The asymptotic behavior of the amplitudes $A_{n}$ given by (56) is

$$
A_{n}(\alpha) \sim \frac{e^{\alpha m}}{n^{m+\frac{1}{2}}} e^{-\omega n\left(\frac{\pi}{2}-\alpha\right)} e^{i \omega n \ln (\omega n)}, \quad n \rightarrow \infty
$$

with $m=\frac{\ln \mu}{\ln \gamma}$. The angular log-frequency $\omega$ is defined by (28). In order to obtain $(57)$, we have used the asymptotic dependence of the $\Gamma$-function asymptote for complex $z$ [39]

$$
\Gamma(z) \simeq e^{(z-1 / 2) \ln z-z}, \quad|z| \gg 1 .
$$

Expression (57) is of the form (21) with $p=m+\frac{1}{2}, \kappa=\omega\left(\frac{\pi}{2}-\alpha\right)$ and $\psi_{n}=\omega n \ln (\omega n)$.

For $\alpha=0$,

$$
A_{n}(0) \sim \frac{1}{n^{m+\frac{1}{2}}} e^{-\frac{\pi}{2} \omega n} e^{i \omega n \ln (\omega n)}, \quad n \rightarrow \infty .
$$

As we have shown above, the fast exponential decay of $A_{n}(0)$ ensures the differentiability of $f(x)$ at all orders. Actually, the fast decay of $A_{n}(0)$ washes out any observable oscillatory structure from the function as seen on figure $2 \mathrm{~d}$. However, there are very tiny log-periodic oscillations of amplitude less than $5 \cdot 10^{-7}$ (see table 1 ) which are however unobservable at the scale of the plot of figure $2 \mathrm{~d}$.

For $\alpha=\pi / 2$ (Weierstrass function), the exponential part disappears and

$$
A_{n}(\pi / 2) \sim \frac{1}{n^{m+\frac{1}{2}}} e^{i \omega n \ln (\omega n)}, \quad n \rightarrow \infty .
$$

This situation corresponds to the case $p=m+\frac{1}{2}$ and $\kappa=0$ and $\psi_{n}=\omega n \ln (\omega n)$ in expression (21) of the classification of section 4.1. The cancellation of the exponential term in $A_{n}$ is due to the very peculiar compensation of the exponential decay of $\Gamma\left(s_{n}\right)$ by the exponential growth of $\cos \left(\alpha s_{n}\right)$ in (53), which occurs only for $\alpha=\frac{\pi}{2}$.

The original Weierstrass function (5) is thus seen as a very special "critical" or bifurcation point of the class of "Weierstrass-type functions" (4) with (50). The analogy goes further as the expression (21) for the amplitudes $A_{n}$ has the same structure as the correlation function of a system of spins where the order $n$ in the sum (18) plays the role of the distance $r$ between two spins. In this analogy, the "correlation length" is proportional to $1 / \kappa \sim\left(\frac{\pi}{2}-\alpha\right)^{-1}$ and diverges at the critical point $\alpha=\frac{\pi}{2}$.

\subsection{Role of the phase: localization and delocalization of singularities}

The phases $\psi_{n}$ defined in (21) play an essential role in the construction of the self-affine nondifferentiable structure of the "Weierstrass-type functions". To stress this fact, let us consider several cases using different phases $\psi_{n}$ with the same absolute values $\left|A_{n}\right|$ of the amplitudes. This study parallels in a sense that of Berry and Lewis [41] and of Hunt [42] but is distinct from it in a essential way as the phases considered here decorate the amplitudes $A_{n}$ in (18) of the power series expansion, rather than the phases in the cosine in (5). Actually, Berry and Lewis study a slight modification of the Weierstrass function (5) defined as

$$
f_{W M}=\sum_{n=0}^{\infty} b^{n}\left(1-\cos \left[a^{n} \pi x\right]\right),
$$


proposed by Mandelbrot [43], which has the property of directly satisfying the "self-affine" property (11) with $\mu=1 / b$ and $\gamma=1$. As discussed above, the choice (54) for $g(x)$, which gives (61) up to a sign, has the advantage of getting rid of the $-1 / s$ correction in its Mellin transform (53) which makes thus more apparent and direct its self-similar properties.

Hunt [42] is able to show that, by replacing the argument $a^{n} \pi x$ of the cosine by $a^{n} \pi x+\theta_{n}$ where $\theta_{n}$ are uncorrelated random phases, the Hausdorff dimension of the phase-randomized Weierstrass function is $D=2-m$.

\subsubsection{Localization of singularities}

Let us first study the case where $\psi_{n}$ is put equal to 0 , i.e., we construct a phase-locked Weierstrass function as

$$
\overline{f_{s}(x)}=\sum_{n=0}^{\infty}\left|A_{n}(\pi / 2)\right| x^{-s_{n}},
$$

i.e., by constructing the singular part as the sum over power laws with amplitudes equal to the modulus of the amplitudes (56) obtained for the Weierstrass function with $\alpha=\pi / 2$, i.e., $\left|A_{n}(\pi / 2)\right|=\left|\frac{\Gamma\left(s_{n}\right) \cos \left((\pi / 2) s_{n}\right)}{\ln \gamma}\right|$, but without the phase. As a consequence, (60) is changed into

$$
\left|A_{n}(\pi / 2)\right|=C \frac{1}{n^{m+\frac{1}{2}}}, \quad \text { for } n \rightarrow \infty
$$

where $C$ is a constant.

Figure 3 shows the function $\overline{f_{s}(x)}$ defined by (62) for $m=0.2$ (panel a) and $m=0.65$ (panel b). Rather than the familiar nondifferentiable self-affine corrugated structure of the Weierstrass function, $\overline{f_{s}(x)}$ seems to be differentiable everywhere except for a discrete infinity of spikes at positions $x_{u}$, where $u$ is an integer running from $-\infty$ to $+\infty$, organized according to a geometric log-periodic structure. This discrete set of spikes decorates the leading singular behavior $f(x) \sim x^{m}$ for $x \rightarrow 0$ of the general solution (7). Note that, in this case, the periodic function $P\left(\frac{\ln x}{\ln \gamma}\right)$ of the general solution (7) is formed by the set of spikes geometrically converging to the origin.

The spikes seem to diverge for $m=0.2$ while they converge to a finite value for $m=0.65$, as far as the numerical construction suggests. Appendix A examines some differentiability properties of (62). Appendix B shows that the functional shapes of the spikes for $x \rightarrow x_{u}=1 / \gamma^{u}$ with $u$ integer are given by

$$
G_{s}(x) \sim \frac{1}{\left|x-x_{u}\right|^{\frac{1}{2}-m}} .
$$

Thus, for $0<m<1 / 2$ (panel (a) of figure 3), the spikes correspond to a divergence of $G_{s}(x)$ as $x \rightarrow x_{u}$. For $1 / 2<m<3 / 2, G_{s}(x)$ goes to a finite value as $x \rightarrow x_{u}$ but with an infinite slope (since $0<m-\frac{1}{2}<1$ ) according to $G_{s}(x) \sim$ constant $-\left|x-x_{u}\right|^{m-\frac{1}{2}}$.

Another example of "localization of singularities" is provided by the function

$$
f_{s}(x)=\sum_{n=1}^{\infty} n^{-m-\frac{1}{2}} e^{i \omega \ln (\omega n)} x^{-s_{n}}
$$

Figure 4 shows this function $f_{s}(x)$ defined by (65) with $m=0.2, \omega=7.7$. One can observe a log-periodic set of structures, each structure composed of log-periodic oscillations converging to singular points beyond which damped oscillation can be observed. Here, the phase $\psi_{n}=\omega \ln (\omega n)$ is not varying fast enough with $n$ to scramble the complex power laws $x^{-s_{n}}$, except at isolated points.

Figure 5 shows the graph of

$$
f_{s}(x)=\sum_{n=1}^{\infty} n^{-m-\frac{1}{2}} e^{i \omega n} x^{-s_{n}}
$$


with $m=0.2, \omega=7.7$ and phases $\psi_{n}=\omega n$. Again, the phase are not sufficiently random to make the function irregular, except at isolated points where the constructive interference of the phases lead to the isolated singularities.

Note that both functions (65) and (66) can be analyzed with the method of Appendix B to obtain the functional form of the singularities.

\subsubsection{Mixing phases}

In contrast to the previous examples where the phases $\psi_{n}$ are too regular, let us now take

$$
\psi_{n}^{(0)}=\omega n \ln (\omega n)
$$

corresponding to the asymptotic dependence (60) of the amplitudes $A_{n}$ of the Weierstrass function. The phases (67) are ergodic and mixing on the unit circle. The corresponding function is

$$
S(x)=\sum_{n=1}^{\infty} n^{-m-\frac{1}{2}} e^{i \omega n \ln (\omega n)} x^{-s_{n}},
$$

which we call the "log-periodic Weierstrass" function to stress the fact that it is constructed by summing log-periodic power laws $x^{-s_{n}}$ (see for instance expression (37)) with amplitudes determined by the asymptotic behavior of the amplitudes of the power expansion of the Weierstrass function itself. The exponents $s_{n}$ are again determined by (15) with $m$ given by (8). By constructing (68), we are stripping off the Weierstrass function of its regular part and of all features that are unrelated to its fundamental nondifferentiability and self-affine properties. The definition of this "log-periodic Weierstrass" function (68) and its many generalizations studied below examplifies the novel construction developed here. $S(x)$ exhibits the same non-differentiability as does the Weierstrass function. In all these cases, $S(x)$ is nondifferentiable since $p=m+\frac{1}{2}<\frac{3}{2}$, in agreement with the conjecture of section 4.1 .

Consider the general case

$$
S_{i}(x)=\sum_{n=1}^{\infty} n^{-m-\frac{1}{2}} \exp \left(i \psi_{n}^{(i)}\right) x^{-s_{n}} .
$$

As other examples, let us now take

$$
\psi_{n}^{(1)}=\omega n^{2}
$$

and

$$
\psi_{n}^{(2)}=\omega e^{n / \omega}
$$

and form the corresponding sums for $i=1$ and $2 . \psi_{n}^{(0)}, \psi_{n}^{(1)}$ and $\psi_{n}^{(2)}$ cause similar irregular oscillations of $\cos \left[\psi_{n}^{(i)}\right]$ between -1 and +1 as a function of $n$, allowing for a non-trivial and complex interactions of singularities whose amplitudes (most important as we have seen) exhibit a slow power-law decay. As the result $S_{1}(x)$ and $S_{2}(x)$ exhibit very clear non-differentiable features shown in figures 9 and 10.

Another very simple example of an ergodic phase is the quadratic rotator with irrational rotation number $0<R<$ $1:$

$$
\psi_{n+1}^{(3)}=\psi_{n}^{(3)}+2 \pi R n .
$$

The most irregular phase is obtained for $R=g=\frac{\sqrt{5}-1}{2}=0.61803398875 \ldots$ which is the golden mean whose main property is that it is the least-well approximated by a rational number. The corresponding "Golden-meanlog-periodic Weierstrass function" $S_{3}^{(g)}(x)$ defined by (69) with (72) is shown in figure 11. Other examples with $R=\pi / 4=0.785398163 \ldots$ and $R=1 / e=0.367879441 \ldots$ lead to $S_{3}^{(\pi / 4)}(x)$ and $S_{3}^{(1 / e)}(x)$ shown in figures 12 and 13. To each irrational number $R$ corresponds an interesting "Weierstrass-type functions" whose delicately corrugated self-affine structure is encoded in the number-theoretical properties of its corresponding irrational number $R$.

Note that if (72) is changed into $\psi_{n+1}^{(3)}=\psi_{n}^{(3)}+2 \pi R$, the corresponding observable $f(x)$ becomes smooth almost everywhere except at isolated points (also organized according to a geometrical series as described in appendix B). The ergodic but non-mixing properties of the linear rotation map does not scramble the phases sufficiently to create non-differentiability. 


\section{$5 \quad$ Illustration of the classification}

Different physical problems will be encoded by different regular parts $g(x)$ quantifying the impact on the observable $f(x)$ of the degrees of freedom summed over between two successive magnifications with the ratio $\lambda$. For a given physical problem, we perform the Mellin transform of $g(x)$. Then, together with the renormalization group structure (2), its formal solution (3) and its expansion (4) close to the leading critical point $x=0$ leading to "Weierstrass-type functions", the classification (21) of the previous section allows us to characterize the possible non-differentiability and scaling properties of the observable $f(x)$.

\subsection{Other examples of the $C^{\infty}$ differentiable family $\kappa>0$}

For a general statistical mechanics model, the regular part $g(x)$ of the free energy has generally the form of the logarithm of a polynomial in $x$. Factorizing the polynomial, we do not lose generality by considering $g(x)$ given by

$$
g(x)=\ln (1+x),
$$

for which

$$
\hat{g}(s)=\frac{\pi}{s \sin s \pi} .
$$

The poles of $\hat{g}$ occur for $s=-n, n>0$, and contribute as already described only to the regular part of $f(x)$. Therefore, the Mellin transform of $f(x)$ is

$$
\widehat{f}(s)=\frac{\mu \gamma^{s}}{\mu \gamma^{s}-1} \frac{\pi}{s \sin (s \pi)} .
$$

The regular part, determined by the poles at $s=-n, n>0$, reads

$$
f_{r}(x)=\sum_{m=1}^{\infty} B(m) x^{m}, \quad B(m)=\frac{(-1)^{m+1}}{m} \frac{\mu}{\mu-\gamma^{m}} .
$$

Note that constant term is absent. The singular part is

$$
f_{s}(x)=\sum_{n=0}^{\infty} A_{n} x^{-s_{n}}, \quad A_{n}=\frac{\pi}{\ln \gamma} \frac{1}{\sin \left(\pi s_{n}\right) s_{n}} .
$$

The coefficients $A_{n}$ converge to zero extremely fast for large $n$

$$
A_{n} \sim \frac{1}{n} e^{-\pi \omega n} e^{-i \pi m}, \quad n \rightarrow \infty
$$

where $\omega$ is given (28). This corresponds to $p=1, \kappa=\pi \omega$ and $\psi_{n}=-\pi m$ in the general classification (21).

For the "lorenzian",

$$
g(x)=\left(1+x^{2}\right)^{-1},
$$

the coefficients $A_{n}$ of the power law expansion (76) of the singular part $f_{s}(x)$ are

$$
A_{n}=\frac{\pi}{2 \ln \gamma} \frac{1}{\sin \left(\pi / 2 s_{n}\right)},
$$

with an asymptotic behavior given by

$$
A_{n} \sim e^{-\frac{\pi}{2} \omega n} e^{i \frac{\pi}{2} m}, \quad n \rightarrow \infty .
$$


This corresponds to $p=0, \kappa=\frac{\pi}{2} \omega$ and $\psi_{n}=\frac{\pi}{2} m$ in the general classification (21). The exponential decay rate $\frac{\pi}{2} \omega$ in this Lorentzian case is half that for the logarithm function (73). Both lead to $C^{\infty}$ differentiable functions with extremely small amplitudes of log-periodic oscillations (see table 1).

For so-called stretched exponential functions

$$
g(x)=e^{-x^{h}}, \quad h>0,
$$

we obtain

$$
A_{n}(h)=\frac{\Gamma\left(s_{n} / h\right)}{\ln (\gamma) h}
$$

and

$$
A_{n}(h) \sim \frac{1}{n^{\frac{m}{h}+\frac{1}{2}}} e^{-\frac{\pi \omega}{2} n} e^{i \frac{\omega n}{h} \ln (\omega n)}, \quad n \rightarrow \infty .
$$

Log-periodic stretched exponential function $f_{s}(x, h)$ and all its derivatives (on $x$ ) converge. This corresponds to $p=\frac{m}{h}+\frac{1}{2}, \kappa=\frac{\pi}{2} \frac{\omega}{h}$ and $\psi_{n}=\frac{\omega n}{h} \ln (\omega n)$ in the general classification (21). Since $\kappa>0$, the corresponding singular function $f_{s}(x)$ is differentiable at all orders. However, a limit of non-differentiability at isolated points can be reached formally by taking the limit $h \rightarrow \infty$ for which $p \rightarrow \frac{1}{2}, \kappa \rightarrow 0^{+}$and and $\psi_{n} \rightarrow 0$. Then, $f_{s}(x)$ exhibits the non-differentiability at points $x_{u}$ verifying $x_{u}=1 / \gamma^{u}$ defined in (106) studied in section 4.4.1. This is shown in figures 14 with the dependence of $f_{s}(x, h)$ on the parameter $h$. As $h$ increases, $g(x)$ becomes more and more localized close to the origin and $f_{s}(x)$ exhibits more and more pronounced steps. Formally, the limit $h \rightarrow \infty$ allows us to cross-over from the class $\kappa>0$ to the class $\kappa=0$.

\subsection{Other examples of the "Weierstrass-type function" class $\kappa=0$}

We have noted above that a non-differentiable function is everywhere oscillating and the length of arc between any two points on the curve is infinite [21]. Its regular generator $g(x)$ must thus contain oscillations or must exhibit at least compact support (so that it has a discrete Fourier series) in order for $f(x)$ to be non-differentiable or for some of its derivatives to be non-differentiable. We illustrate this remark by several examples.

\subsubsection{Generalized periodic processes}

Let us consider the function generalizing the sinc-function by taking an arbitrary real exponent $\delta$ :

$$
g(t)=\frac{\sin (t)}{t^{\delta}} .
$$

The Weierstrass function is recovered for $\delta=0$. The coefficients $A_{n}$ of the expansion in power series of the singular part can be obtained by a simple shift of $s$ in the expression obtained for the Weierstrass function:

$$
A_{n}(\delta)=\frac{\Gamma\left(s_{n}-\delta\right) \sin \left(\frac{1}{2} \pi\left(s_{n}-\delta\right)\right)}{\ln \gamma}
$$

For $m+\delta \leq 1$, the log-periodic generalized sinc-function $f_{s}(x, \delta)$ as well as its associated Weierstrass-type function are continuous but non-differentiable.

The asymptotic behavior of the coefficient $A_{n}$ is

$$
A_{n}(\delta) \sim n^{-m-\delta-1 / 2} e^{-i \omega n \ln (\omega n)}, \quad n \rightarrow \infty .
$$

This corresponds to $p=m+\delta+1 / 2, \kappa=0$ and $\psi_{n}=\omega n \ln (\omega n)$ in the general classification (21). Figure 15 shows the generalized sinc-function $f_{s}(x, \delta)$ for different values of $\delta$ with for $\delta=-0.1$ (solid line), $\delta=0$ (dashed line) and $\delta=0.1$ (dotted line) for $m=0.4$ and $\omega=7.7$. 
Another interesting case is the sine integral, $g(x)=S i(x) \equiv \int_{0}^{x} d v \frac{\sin v}{v}$. The coefficients $A_{n}$ of the power law series of the singular part are given by

$$
A_{n}=-\frac{\Gamma\left(s_{n}\right) \sin \left(\frac{1}{2} \pi s_{n}\right)}{\ln \gamma s_{n}}
$$

with asymptotics

$$
A_{n} \sim n^{-m-3 / 2} e^{-i \omega n \ln (\omega n)+\frac{\pi}{2} m}, \quad n \rightarrow \infty,
$$

corresponding to $p=m+3 / 2, \kappa=0$ and $\psi_{n}=\omega n \ln (\omega n)+\frac{\pi}{2} m$ in the general classification (21). All three functions $f(x), f_{s}(x)$ and $S(x)$ defined by (68) have a continuous but non-differentiable first derivative for $m<1$. However, the delicate log-periodic corrugations are enhanced in the graph of $S(x)$.

\subsubsection{Localized processes}

Let us now study functions $g(x)$ with compact support such as

$$
g(x)=\left(1-x^{h}\right)^{\nu-1}, \quad 0 \leq x \leq 1, \quad g(x)=0 \quad \text { for } x>1 \text { with } h \geq 1 \text { and } \nu \geq 2 .
$$

The coefficients $A_{n}$ of the power series expansion of the singular part $F_{s}(x)$ are

$$
A_{n}(\nu, h)=\frac{B\left(\nu, s_{n} / h\right)}{\ln (\gamma) h},
$$

where $B(x, y)=\frac{\Gamma(x) \Gamma(y)}{\Gamma(x+y)}$ is the beta-function. Figure 17 shows the function $f(x)$ obtained from the direct sum (凹). Figure 18 shows how the shape of the log-periodical structures steepen with increasing $h$, as the function $g(x)$ evolves from a half- $\cap$ shape to a the plateau $g(0<x<1)=1$ and 0 otherwise. The log-periodic geometrical series of plateaux and steps shown in figure 18 is reminiscent of the structures found for rupture [28, 29, 5] and earthquakes [6, 30] precursors.

The asymptotic behavior of $A_{n}(\nu)$ is

$$
A_{n}(\nu, h) \sim \frac{e^{i \pi}}{n^{\nu}}, \quad \text { with } \nu \geq 2, \quad n \rightarrow \infty .
$$

corresponding to $p=\nu, \kappa=0$ and $\psi_{n}=\pi$ in the general classification (21).

As the phases $\psi_{n}=\pi$ are constant and their contribution can be factorized, the function $f_{s}(x, \nu)$ has a behavior similar to the function (52) analyzed in section 4.4.1. In particular, we recover the fact that the points $x_{u}=1 / \gamma^{u}$ given by (106) make the imaginary contribution of $x^{-s_{n}}$ vanish. As a consequence, they are the most singular points. An analysis similar to that presented in section 4.4.1 can be performed.

Another example corresponds to the logarithmic function

$$
g(x)=\ln (1-x), \quad 0<x<1 \quad \text { and } g(x)=0 \text { for } x>1,
$$

with compact support. Figure 19 shows the corresponding Weierstrass function $(m=0.5, \omega=7.7, N=47)$. The coefficients in the power expansion are given by

$$
A_{n}=-\frac{1}{s_{n}}\left[\Psi\left(1+s_{n}\right)-\Psi(1)\right]
$$

where $\Psi$ is the logarithmic derivative of the Gamma function.

The asymptotic behavior of the coefficients $A_{n}$ is

$$
A_{n} \sim \frac{\ln (n)}{n} \exp \left[i\left(-\arctan \left(\frac{2 \ln (\omega n)}{\pi}\right)+\pi\right)\right],(n \rightarrow \infty),
$$


corresponding to $p=1, \kappa=0$ and $\psi_{n}=\arctan \left(\frac{2 \ln (\omega n)}{\pi}\right)$ in the general classification (21). The logarithmic "correction" to the power law $1 / n$ comes from the singularity at $x=1$. This example illustrates a possible cause for a deviation from the classification (21). Such modification however does not change the qualitative picture as they correspond to the next sub-dominant correction to the power law contribution to $A_{n}$.

The relative amplitudes of the two first power law terms are given by $\left|\frac{A_{n=1}}{A_{n=0}}\right|=0.143, \quad\left|\frac{A_{n=2}}{A_{n=0}}\right|=0.086, \quad(m=$ $0.5, \omega=7.7)$.

For the non-singular compact logarithmic regular part

$$
g(x)=\ln (1+x), \quad 0<x<1 \quad \text { and } g(x)=0 \text { for } x>1,
$$

figure 21 shows the corresponding Weierstrass-type function $f(x)$. The coefficients in the power series expansion are

$$
A_{n}=\frac{1}{s_{n}}\left[\log (2)-\frac{1}{2} \Psi\left(1+\frac{s_{n}}{2}\right)+\frac{1}{2} \Psi\left(\frac{1}{2}+\frac{s_{n}}{2}\right)\right],
$$

where $\Psi$ is again the logarithmic derivative of the Gamma function. The asymptotic behavior of the coefficients $A_{n}$ is

$$
A_{n} \sim \frac{1}{n} \exp \left[i\left(\arctan \left(\frac{\omega n}{m}\right)+\pi\right)\right],(n \rightarrow \infty),
$$

corresponding to $p=1, \kappa=0$ and $\psi_{n}=\arctan \left(\frac{\omega n}{m}\right)+\pi$ in the general classification (21). The relative amplitudes of the two first power law terms are given by $\left|\frac{A_{n=1}}{A_{n=0}}\right|=0.016,\left|\frac{A_{n=2}}{A_{n=0}}\right|=7.73810^{-3}$ for $m=0.5, \omega=7.7$. Thus, even if the asymptotic decay is almost the same as for (93) up to the logarithmic correction, the logperiodic amplitudes of the leading terms are a factor of 10 smaller.

\section{Discussion}

This paper has studied the solutions of the equation ( $\bigoplus$, which can be understood as a renormalization group equation with a single control parameter or more generally as the Jackson $q$-integral describing discrete scale invariant (DSI) systems. We have put the emphasis on the factors controlling the presence and amplitude of log-periodic corrections to the leading power law solution. We have used the Mellin transform to resum the formal series solution of the DSI equation into a power law series and have presented a general classification within two classes:

1. systems with quasi-periodic "regular part" and/or with compact support present strong log-periodic oscillatory amplitudes.

2. systems with non-periodic "regular part" with unbound support have exceedingly small log-periodic oscillatory amplitudes and regular smooth observables.

In systems for which the renormalization group equation has been explicited, systems of the first class are associated with "antiferromagnetic" interactions. Systems of the second class occur when the microscopic interactions are dominantly "ferromagnetic". These facts plus the observation that strong log-periodic oscillations have been reported in out-of-equilibrium growth processes, in rupture, in earthquakes and in finance lead us to propose that strong logperiodicity is associated with the presence of interactions with competing signs and long-range dipolar and multipolar form, favoring different "signs" and modulated patterns. Dipolar and/or antiferromagnetic interactions are well-known to lead to a rich behavior with striped patterns with alternating up and down spins (see [45] and references therein) which are robust in the presence of disorder such as in the dipolar random-field Ising model [46, 47]. We conjecture that these spatial organization translates into a quasi-periodic "regular part" (or with compact support) of the renormalization group description.

The existence of "antiferromagnetic" or "dipolar" interactions leading to coexistence of different "signs" and of modulated structures is documented in rupture and finance. In rupture phenomena, cracking or local breaking leads 
to a redistribution of stress according to the elastic "dipolar" Green function which is non-monotonous and exhibit oscillations as a function of distance with local enhancement as well as screening alternating as a function of angular position. Similarly, an earthquake (corresponding technically to a "double-couple" force source within the elastic crust) redistributes stress according to a clover-leaf pattern with alternating + and - signs of the stress as a function of angular position. This superficial analogy has been shown to be quite precise in a mapping between the random fuse model and a dipolar magnet in which the state of the network damage is associated with a metastable spin configuration 48 .

Similarly, the complex price time series observed in financial stock markets can be seen to result from the competition between "value-searching" investors [49, 50] who track the fundamental value of the stocks and "trend-following" traders (see [51] and references therein) who imitate the crowd thus developing herding behavior. Roughly speaking, the former (later) type of traders sell (buy) when the price increases above the fundamental value and sell (buy) when the reverse occurs. Thus, "value-searching" investors follow interactions similar to "antiferromagnetic" coupling while the herding behavior resulting from the action of "trend-following" investors can be captured quantitatively by "ferromagnetic"-type interactions.

We believe that the existence of non-monotonous interactions with competing signs may be a fundamental mechanism of strong log-periodicity. Our analysis of its impact on the "regular part" of the DSI equation suggests that the "antiferromagnetic" and/or "dipolar" interactions are crucial ingredients at the origin of fractal patterns in nature.

However, determining the observables that take the place of the equilibrium free energy for growth models which could be obtained recursively (via a renormalisation group transformation) - or equivalently, the identification of the meaning of the function $g(x)$, remains an unsolved problem. Correlatively, the mechanisms in growth models that select the log-period of the observed log-periodic oscillations in the absence of a hierarchical lattice have not been fully elucidated (see however [ $₫$ ] for a specific needle growth model and [1] for a review). In a way, our paper has attempted to provide an explanation of why the log-periodic oscillations may have a strong amplitude, without explaining really their origin! As a consequence, the program ahead of us is i) to identify clearly the mechanism(s) underlying the emergence of DSI, ii) derive the equivalent renormalization group equation. Only then, shall we be able to substantiate convincingly our conjectured mechanism in terms of "anti-ferromagnetic" interactions.

Acknowledgments: We are grateful to A. Erzan for a discussion on Jackson's integral and for supplying the correponding references and to the referees' suggestions that helped us improve the manuscript. 


\section{Appendix A: Differentiability properties of the "localization of singularities"}

Using the asymptotic expression (63), we can write

$$
\operatorname{Re}\left[\overline{f_{s}(x)}\right]=G(x)+x^{m} \sum_{n=n_{r}}^{\infty} \frac{1}{n^{m+\frac{1}{2}}} \cos \left(2 \pi n \frac{\ln x}{\ln \gamma}\right),
$$

where $G(x)=\sum_{n=0}^{n_{r}}\left|A_{n}(\pi / 2)\right| x^{-s_{n}}$ is a regular function. $\operatorname{Re}\left[\overline{f_{s}(x)}\right]$ denotes the real part of $\overline{f_{s}(x)}$ and we have used (15). The second term of the r.h.s. of (99), which can be called the singular part of $\operatorname{Re}\left[\overline{f_{s}(x)}\right]$ and is denoted $G_{s}(x)$, is a sum starting at an index $n_{r}$ which is taken sufficiently large such that the asymptotic expression (63) holds to within any desired degree of accuracy.

The singular part $G_{s}(x)$ has the same analytical behavior as the function

$$
K_{p}(y) \equiv \sum_{n=1}^{\infty} \frac{1}{n^{p}} \cos (n y), \quad p=m+\frac{1}{2},
$$

where $y=2 \pi \ln x / \ln \gamma$. This function is a special case of $K_{p,\left\{\psi_{n}\right\}}(y)$ defined by (41) for $\psi_{n}=0$.

This function $K_{p}(y)$ has been studied in the literature for special cases. When the real part of $p$ is larger than 1 , the sum is absolutely convergent for all $x$. Restricting our attention to real exponents $p$, the series $K_{p}(y=\pi)=$ $-\sum_{n=1}^{\infty} \frac{(-1)^{n+1}}{n^{p}}$, which corresponds to $y=(2 \ell+1) \pi$ where $\ell$ is an arbitrary integer, is convergent for all positive $p$ to $K_{p}(y=\pi)=\left(1-2^{1-p}\right) \zeta(p)$ [40], where $\zeta(p)$ is the Riemann zeta function. Obviously, $K_{p}(y=2 \pi)$ is infinite for $p<1$ and we show below that $K_{p}(y \rightarrow 2 \pi)$ has a power law singularity. For $p>1, K_{p}(y)$ can be expressed as

$$
K_{p}(y)=\frac{(2 \pi)^{p}}{4 \Gamma(p)} \sec \left(\frac{\pi p}{2}\right)\left[\zeta\left(1-p, \frac{y}{2 \pi}\right)-\zeta\left(1-p, 1-\frac{y}{2 \pi}\right)\right]
$$

where $\zeta(s, \nu)=\sum_{k=0}^{+\infty}[\nu+k]^{-s}$ is the generalized Riemann zeta function [44].

For $p>0$ and except for the special value $y=0$ modulus $2 \pi, K_{p}(y)$ is finite and differentiable. To see this, let us consider rational values of $y / 2 \pi=r / q$ with $q \geq 2$, where $r / q$ is the irreducible representation of the rational $y / 2 \pi$. We can rearrange the series in (100) into $q$ sub-series as follows:

$$
\begin{aligned}
K_{p}(y) & =\operatorname{Re}\left(\sum_{k=1}^{\infty} \frac{1}{(k q)^{p}}+\sum_{k=1}^{\infty} \frac{e^{i 2 \pi(q-1)(r / q)}}{(k q-1)^{p}}+\sum_{k=1}^{\infty} \frac{e^{i 2 \pi(q-2)(r / q)}}{(k q-2)^{p}}+\ldots\right. \\
& \left.+\sum_{k=1}^{\infty} \frac{e^{i 2 \pi 2(r / q)}}{(k q-q+2)^{p}}+\sum_{k=1}^{\infty} \frac{e^{i 2 \pi(r / q)}}{(k q-q+1)^{p}}\right)=\operatorname{Re} \sum_{j=1}^{q}\left(\sum_{k=0}^{\infty} \frac{e^{i 2 \pi j(r / q)}}{(k q+j)^{p}}\right) \\
& =\operatorname{Re} \sum_{k=0}^{\infty}\left(\sum_{j=1}^{q} \frac{e^{i 2 \pi j(r / q)}}{(k q+j)^{p}}\right),
\end{aligned}
$$

We expand $1 /(k q+j)^{p}=(k q)^{-p}\left[1-\frac{p}{k q} j+\frac{p(p+1)}{2} \frac{j^{2}}{(k q)^{2}}+\ldots\right]$ and get

$$
K_{p}(y / 2 \pi=r / q)=\operatorname{Re} \sum_{k=0}^{\infty} \frac{1}{(k q)^{p}}\left(\sum_{j=1}^{q} e^{i 2 \pi j(r / q)}-\frac{p}{k q} \sum_{j=1}^{q} j e^{i 2 \pi j(r / q)}+\frac{p(p+1)}{2(k q)^{2}} \sum_{j=1}^{q} j^{2} e^{i 2 \pi j(r / q)}+\ldots\right) .
$$

Calling $w_{q}$ the $q$ th root of 1 , i.e., $w_{q}=e^{i 2 \pi / q}$, we have then $w_{q}+w_{q}^{2}+\ldots+w_{q}^{q-1}+w_{q}^{q}=\left(1-w_{q}^{q}\right) /(1-w)=0$. Hence, the first sum $\sum_{j=1}^{q} e^{i 2 \pi j(r / q)}$ in (103) is identically zero. The other sums are non zero and finite. We thus get

$$
K_{p}(y / 2 \pi=r / q)=\sum_{k=0}^{\infty} \frac{C_{k}(r, q)}{k^{p+1}}
$$


where

$$
C_{k}(r, q)=\frac{1}{q^{p+1}} \operatorname{Re}\left[\sum_{j=1}^{q} e^{i 2 \pi j(r / q)}\left(-p j+\frac{p(p+1)}{2(k q)} j^{2}-\frac{p(p+1)(p+2)}{6(k q)^{2}} j^{3}+\ldots\right)\right]
$$

is bounded from above as $k \rightarrow+\infty$. The expression (104) shows that $K_{p}(y / 2 \pi=r / q)$ is finite for any $p>0$. Now, since rational numbers are dense among real numbers, i.e., any irrational number can be approached arbitrarily close by a rational number, by the condition of continuity, $K_{p}(y)$ is finite everywhere, except for $y / 2 \pi=r / q$ with $q=1$. Differentiating the expression (100) gives the series $\sum_{n=1}^{\infty} \frac{1}{n^{p-1}} \sin (n y)$. By the same reasoning leading to (104), this derivative is bounded from above by a constant times $\sum_{n=1}^{\infty} \frac{1}{n^{p}}$ which is convergent for $p>1$. This shows that $K_{p}(y)$ is differentiable for $p>1$, and thus $\operatorname{Re}\left[\overline{f_{s}(x)}\right]$ is differentiable for $m>1 / 2$. This approach is not powerful enough however to treat the case $m<1 / 2$. 


\section{APPENDIX B: Functional shape of the denumerable set of discrete singularities re- sulting from the "localization of singularities"}

We now examine the special case $y / 2 \pi=r / q$ with $q=1$. From the expression (99), the values $x_{u}$, which are such that $\ln x_{u} / \ln \gamma$ is an integer $-u$, i.e.,

$$
x_{u}=1 / \gamma^{u},
$$

make all the cosine terms in the infinite sum in phase and equal to 1 . Thus,

$$
G_{s}\left(x_{u}\right)=x^{m} \sum_{n=n_{r}}^{\infty} \frac{1}{n^{m+\frac{1}{2}}}
$$

which diverges for $m \leq 1 / 2$. At the border case $m=1 / 2$, the divergence is logarithmic. Similarly, $d G_{s} /\left.d x\right|_{x=x_{u}}$ diverges for $m<3 / 2$ as an additional power of $n$ is brought to each term in the sum by taking the derivative. This and expression (106) explain the graphs of Figure 3.

The functional shapes of the spikes for $x \rightarrow x_{u}$ can be determined as follows. For $x \rightarrow x_{u}, \cos \left(2 \pi n \frac{\ln x}{\ln \gamma}\right)=$ $\cos \left(\frac{2 \pi n \epsilon}{\ln \gamma}\right)+\mathcal{O}\left(\epsilon^{2}\right)$, where $\epsilon \equiv\left(x-x_{u}\right) / x_{u}$ and $\mathcal{O}\left(\epsilon^{2}\right)$ represents a term proportional to $\epsilon^{2}$. Let us now construct and compare $G_{s}(\epsilon)$ and $G_{s}(\lambda \epsilon)$, where $\lambda$ is an arbitrary number. Up to first order in $\epsilon$, we have

$$
G_{s}(\lambda \epsilon) \approx x_{u}^{m} \sum_{n=n_{r}}^{\infty} \frac{\cos \left(\frac{2 \pi n \lambda \epsilon}{\ln \gamma}\right)}{n^{m+\frac{1}{2}}} .
$$

Posing $n^{\prime}=\operatorname{Int}(n \lambda), G_{s}(\lambda \epsilon)$ can be rewritten

$$
G_{s}(\lambda \epsilon) \approx x_{u}^{m} \sum_{n^{\prime}=\operatorname{Int}\left[n_{r} \lambda\right]}^{\infty} \frac{\lambda^{m+\frac{1}{2}}}{\lambda} \frac{\cos \left(\frac{2 \pi n^{\prime} \epsilon}{\ln \gamma}\right)}{n^{\prime m+\frac{1}{2}}}=x_{u}^{m} \lambda^{m-\frac{1}{2}} \sum_{n^{\prime}=\operatorname{Int}\left[n_{r} \lambda\right]}^{\infty} \frac{\cos \left(\frac{2 \pi n^{\prime} \epsilon}{\ln \gamma}\right)}{n^{\prime m+\frac{1}{2}}} .
$$

Note the presence of the additional multiplicative term $\frac{\lambda^{m+\frac{1}{2}}}{\lambda}$ in the sum of (109). The numerator $\lambda^{m+\frac{1}{2}}$ stems from replacing $n$ by $n^{\prime}=\operatorname{Int}(n \lambda)$ in $1 / n^{m+\frac{1}{2}}$. The other factor $1 / \lambda$ is the "Jacobian" of the change from $n$ to $n^{\prime}=\operatorname{Int}(n \lambda)$. Expression (109) can then be rewritten

$$
G_{s}(\lambda \epsilon) \approx \lambda^{m-\frac{1}{2}} G_{s}(\epsilon)+H_{r}(\epsilon)
$$

where

$$
H(\epsilon)=\lambda^{m-\frac{1}{2}} \sum_{n=n_{r}}^{\operatorname{Int}\left[n_{r} \lambda\right]-1} \frac{\cos \left(\frac{2 \pi n^{\prime} \epsilon}{\ln \gamma}\right)}{n^{\prime m+\frac{1}{2}}}
$$

is a regular function of $\epsilon$. The singular part of $G_{s}(\epsilon)$ is solution of $G_{s}(\lambda \epsilon) \approx \lambda^{m-\frac{1}{2}} G_{s}(\epsilon)$, i.e.,

$$
G_{s}(\epsilon) \sim \epsilon^{m-\frac{1}{2}} .
$$

This confirms that, for $0<m<1 / 2$ (panel (a) of figure 3), the spikes correspond to a divergence of $G_{s}(x)$ as $x \rightarrow x_{u}$ according to $G_{s}(x) \sim 1 /\left|x-x_{u}\right|^{\frac{1}{2}-m}$. For $1 / 2<m<3 / 2, G_{s}(x)$ goes to a finite value as $x \rightarrow x_{u}$ but with an infinite slope (since $0<m-\frac{1}{2}<1$ ) according to $G_{s}(x) \sim$ constant $-\left|x-x_{u}\right|^{m-\frac{1}{2}}$. The borderline case $m=1 / 2$ can actually be summed exactly as $K_{p=1}(y)=-\frac{1}{2} \ln (2[1-\cos y])$ [40]. When $y \rightarrow 0$ modulo $2 \pi, K_{p=1}(y)$ diverges as $\ln \frac{1}{y}$ and thus $G_{s}(\epsilon)$ diverges as $G_{s}(\epsilon) \sim \ln \left|\frac{x_{u}}{x-x_{u}}\right|$. 


\section{References}

[1] D. Sornette, Discrete Scale Invariance and Complex Dimensions, Physics Reports 297, 239-270 (1998).

[2] B. Derrida, C. Itzykson and J.M. Luck, Oscillatory critical amplitudes in hierarchical models, Commun. Math. Phys. 94, 115-132 (1984).

[3] D. Sornette, A. Johansen, A. Arnéodo, J.-F. Muzy and H. Saleur, Complex fractal dimensions describe the internal hierarchical structure of DLA, Phys. Rev. Lett. 76, 251-254 (1996).

[4] Y. Huang, G. Ouillon, H. Saleur and D. Sornette, Spontaneous generation of discrete scale invariance in growth models, Physical Review E 55, N6, 6433-6447 (1997).

[5] A. Johansen and D. Sornette, Critical ruptures, Eur. Phys. J. B 18, 163-181 (2000).

[6] D. Sornette and C.G. Sammis, Complex critical exponents from renormalization group theory of earthquakes: Implications for earthquake predictions, J.Phys.I France 5, 607-619 (1995).

[7] D. Sornette, A. Johansen and J.-P. Bouchaud, Stock market crashes, Precursors and Replicas, J.Phys.I France 6, 167-175 (1996).

[8] A. Johansen, D. Sornette and O. Ledoit, Predicting Financial crashes using discrete scale invariance, Journal of Risk 1, 5-32 (1999).

[9] F.H. Jackson, Q.J. Pure. Appl. Math 41, 193 (1910); Q.J. Math Oxford Ser. 2, 1 (1951).

[10] A. Erzan, Finite $q$-differences and the discrete renormalization group, Phys. Letts. A 225, 235-238 (1997).

[11] A. Erzan and J.-P. Eckmann, $q$-analysis of Fractal Sets, Phys. Rev. Letts. 78, 3245-3248 (1997).

[12] B. Derrida, J-P Eckmann and A. Erzan, Renormalization groups with periodic and aperiodic orbits, J. Phys. A, 16, 893-906 (1983).

[13] S. R. McKay, A.N. Berker and S. Kirkpatrick, Spin-glass behavior in frustrated Ising models with chaotic renormalization group trajectories, Phys. Rev. Letts. 48, 767-770 (1982).

[14] G. Paul, Coefficient scaling, Phys. Rev. E 59, 4847-4856 (1999).

[15] B. Dubrulle, F. Graner and D. Sornette, eds., Scale invariance and beyond, (EDP Sciences and Springer, Berlin, 1997).

[16] D. Sornette, Critical Phenomena in Natural Sciences (Chaos, Fractals, Self-organization and Disorder: Concepts and Tools) (Springer Series in Synergetics, 2000).

[17] N. Goldenfeld, Lectures on phase transitions and the renormalization group (Reading, Mass.: Addison-Wesley, Advanced Book Program, 1992).

[18] A. Erzan, Phys. Lett. A 225, 235 (1997).

[19] K. Weierstrass, On Continuous Functions of a Real Argument that do not have a Well-defined Differential Quotient, in Classics on Fractals, Edited by G. A. Edgar (Addison-Wesley, Reading, Massachusets, 1993) p. 3-9.

[20] G.H. Hardy, Weierstrass's non-differentiable function, Trans. Amer. Math. Soc. 17, 301-325 (1916). 
[21] A.N. Singh, The theory and construction of non-differentiable functions, in Squaring the circle and other monographs (Chelsea Publishing Company, 1953).

[22] L. F. Richardson, Atmospheric Diffusion on a Distance-Neighbour Graph, Proc. Roy. Soc. London A 110, 709 (1926).

[23] B. D. Hughes, M.F. Shlesinger, and E. W. Montroll, Random walks with self-similar clusters, Proc. Natl. Acad. Sci. U.S.A. 78, $3287-3291$ (1981); E.W. Montroll and M. F. Shlesinger, in Studies in Statistical Mechanics, edited by J. Lebowitz and E. Montroll (North-Holland, Amsterdam, 1984), Vol. 11, p. 1; J. Klafter, $\mathbf{M}_{i} \mathrm{~F}_{i}$ Shlesinger and G. Zumofen, Beyond Brownian motion, Physics Today 49, 33-39 (1996); M.F. Shlesinger, G.M. Zaslavsky and J. Klafter, Strange kinetics, Nature 363, 31-37 (1993).

[24] H. Saleur and D. Sornette, Complex exponents and log-periodic corrections in frustrated systems, J.Phys.I France 6, 327-355 (1996).

[25] M.L. Lapidus and M. van Frankenuysen, Fractal geometry and number theory (Birchäuser, Boston, Basel, Berlin, 2000).

[26] Yu.V. Sidorov, M. V. Fedoryuk and M. I. Shabunin, Lectures in Theory of Complex Variable, Nauka, Moscow (1989), p.480.

[27] F. Oberhettinger, Tables of Mellin Transforms, Springer-Verlag, N.Y. (1974), p.275

[28] J.-C. Anifrani, C. Le Floc'h, D. Sornette and B. Souillard, Universal Log-periodic correction to renormalization group scaling for rupture stress prediction from acoustic emissions, J.Phys.I France 5, 631-638 (1995).

[29] A. Johansen and D. Sornette, Evidence of discrete scale invariance by canonical averaging, Int. J. Mod. Phys. C 9, 433-447 (1998).

[30] H. Saleur, C.G. Sammis and D. Sornette, Discrete scale invariance, complex fractal dimensions and log-periodic corrections in earthquakes, J. Geophys. Res. 101, 17661-17677 (1996).

[31] A. Johansen, H. Saleur and D. Sornette, New Evidence of Earthquake Precursory Phenomena in the 17 Jan. 1995 Kobe Earthquake, Japan, Eur. Phys. J. B 15, 551-555 (2000).

[32] A. Johansen and D. Sornette, The Nasdaq crash of April 2000: Yet another example of log-periodicity in a speculative bubble ending in a crash, Eur. Phys. J. B 17, 319-328 (2000).

[33] R. Metzler, J. Klafter and J. Jortner, Hierarchies and logarithmic oscillations in the temporal relaxation patterns of proteins and other complex systems, Proc. Natl. Acad. Sci. USA 96, 11085-11089 (1999).

[34] T.-Y. Hu and K.-S. Lau, Fractal dimensions and singularities of the Weierstrass-type functions, Trans. Am. Math. Soc. 335, 649-665 (1993).

[35] E.C. Titchmarsh, The Zeta-Function of Riemann, Cambridge University Press, London, 1930, p. 104.

[36] H.M. Edwards, Riemann's zeta function, Academic Press, New York (1974).

[37] J.L. Kaplan, J. Mallet-Paret and J.A. Yorke, The Lyapunov dimension of a nowhere differentiable attracting torus, Ergod. Th. Dynam. Sys. 4, 261-281 (1984).

[38] Tables of integral transforms, Edited by. A. Erde' lyi, McGraw-Hill, New York, 1954.

[39] N.N. Lebedev, Special functions and their applications, Englewood Cliffs, N. J., Prentice-Hall, 1965, p.308. 
[40] I. S. Gradshteyn and I.M. Ryzhik, Table of integrals, series, and products, 4th ed. prepared by Yu. V. Geronimus and M. Yu. Tseytlin, Translated from the Russian by Scripta Technika, inc. Translation edited by Alan Jeffrey (New York, Academic Press, 1965).

[41] M. V. Berry and Z. V. Lewis, On the Weierstrass-Mandelbrot fractal function, Proc. Roy. Soc. A 370, 459-484 (1980).

[42] B.R. Hunt, The Hausdorff dimension of graphs of Weierstrass functions, Proc. Am. Math. Soc 126, 791-800 (1998).

[43] B. Mandelbrot, The fractal Geometry of Nature (Freeman, San Francisco, 1982).

[44] A.P. Prudnikov, Yu. A. Brychkov, O.I. Marichev, Integrals and series, translated from the Russian by N.M. Queen (New York: Gordon and Breach Science Publishers, 1986-1992).

[45] K. De'Bell, Maclsaac, A.B. and Whitehead, J.P., Dipolar effects in magnetic thin films and quasi-twodimensional systems, Rev. Mod. Phys. 72, 225-257 (2000).

[46] T. Natterman, Dipolar interaction in random-field systems, J. Phys. A: Math. Gen. 21, L645-L649 (1988).

[47] A. Magni, Hysteresis properties at zero temperature in the dipolar random-field Ising model, Phys. Rev. B 59, 985-990 (1999).

[48] M. Barthélémy, R. da Silveira and H. Orland, The random fuse network as a dipolar magnet, cond-mat/0106012

[49] Bouchaud, J.-P. and R. Cont (1998) A Langevin approach to stock market fluctuations and crashes, Eur. Phys. J. B 6, 543-550.

[50] K. Ide and D. Sornette, Oscillatory Finite-Time Singularities in Finance, Population and Rupture, preprint (eprint at http://arXiv.org/abs/cond-mat/0106047)

[51] Andersen, J.V., S. Gluzman and D. Sornette (2000) Fundamental Framework for Technical Analysis, European Physical Journal B 14, 579-601. 


\begin{tabular}{|c|c|c|c|c|c|}
\hline$g(x)$ & $p$ & $\kappa$ & $\psi_{n}$ & $\frac{A_{n=1}}{A_{n=0}}$ & $\frac{A_{n=2}}{A_{n=0}}$ \\
\hline $\cos (x)$ & $m+1 / 2$ & 0 & $\omega n \ln (\omega n)$ & 0.065 & 0.032 \\
\hline $\exp (-x)$ & $m+1 / 2$ & $\frac{\pi}{2} \omega$ & $\omega n \ln (\omega n)$ & $5.1210^{-7}$ & $1.43210^{-12}$ \\
\hline $\exp [-c x] \cos (x s)^{*}$ & $m+1 / 2$ & $\left.\frac{\pi}{2}-\alpha\right) \omega$ & $\omega n \ln (\omega n)$ & & \\
\hline$\left(1+x^{2}\right)^{-1}$ & 0 & $\frac{\pi}{2} \omega$ & $\frac{\pi}{2} m$ & $9.90110^{-6}$ & $4.41410^{-11}$ \\
\hline $\log (1+x)$ & 1 & $\pi \omega$ & $-\pi m$ & $4.04510^{-12}$ & $\approx 0$ \\
\hline $\exp \left(-x^{h}\right)$ & $m / h+1 / 2$ & $\frac{\pi}{2 h} \omega$ & $\frac{\omega n}{h} \ln (\omega n)$ & $0.064(h=50)$ & $0.03(h=50)$ \\
\hline & & & & $4.38610^{-4}(h=2)$ & $6.17710^{-7}(h=2)$ \\
\hline $\sin (x)$ & $m+\delta+1 / 2$ & 0 & $-\omega n \ln (\omega n)$ & $0.044(\delta=0.1)$ & $0.021(\delta=0.1)$ \\
\hline$x^{\delta}$ & & & & $0.091(\delta=-0.1)$ & $0.049(\delta=-0.1)$ \\
\hline$S i(x)$ & $m+3 / 2$ & 0 & $\omega n \ln (\omega n)$ & $4.19910^{-3}$ & $1.05310^{-3}$ \\
\hline $1-x^{h} 0<x<1$ & 2 & 0 & $\pi$ & $0.064(h=50)$ & $0.031(h=50)$ \\
\hline & & & & $0.012(h=2)$ & $3.14610^{-3}(h=2)$
\end{tabular}

Table 1: Synthesis of the different classes of Weierstrass-type functions according to the general classification (21) $A_{n} \sim \frac{1}{n^{p}} e^{-\kappa n} e^{i \psi_{n}}$ of the expansion (18) in terms of a series of power laws $x^{-s_{n}}$. The parameters $p, \kappa \geq 0$ and $\psi_{n}$ are determined by the form of $g(x)$ and the values of $\mu$ and $\gamma$. All numerical values given in this table correspond to $m=0.5, \omega=7.7$ corresponding to $\gamma=2.26$ and $\mu=\sqrt{\gamma}=1.5$. The last two columns quantify the amplitude of the log-periodic oscillations with respect to the leading real power law. $(*) c=\cos \alpha$ and $s=\sin \alpha$. 


\section{FIGURE CAPTIONS}

FIGURE 1: Power law expansion part $f_{s}$ given by (18) for the Weierstrass function (5), with $N=1$ (solid), $N=2$ (dash), $N=3$ (dot) oscillatory terms, respectively. Here, $m=0.25, \omega=6.3$ corresponding to $\gamma=2.7$ and $\mu=1.28$. As the number of complex exponents increases, the number of the oscillations increase.

FIGURE 2: Quasi-Weierstrass function for (a) $\alpha=\frac{\pi}{2}$, (b) $\alpha=0.993 \pi / 2=1.56$, (c) $\alpha=0.9 \pi / 2=1.414$ and (d) $\alpha=0$, for $m=0.25, \omega=7.7$, using $N=32$ terms to estimate the sums (51). Increasing $N$ does not change the results

FIGURE 3: Panels (a) and (b) show $f_{s}(x)$ defined by (62) with zero phase $\psi_{n}=0$, for $m=0.2$ and $m=0.65$ respectively, with the same $\omega=7.7$, constructed by truncating the sum at the $N=29$ term. The tiny regular oscillations result from the truncation to a finite $N$ and slowly vanish when $N \rightarrow \infty$. They are thus spurious finitesize effects.

FIGURE 4: Graph of $f_{s}(x)$ defined by (65) with $\psi_{n}=\omega \ln (\omega n)$, with $m=0.2, \omega=7.7$ and using $N=1000$ terms in the sum.

FIGURE 5: Graph of shows $\mathrm{f}_{s}(x)$ defined by (66) with $\psi_{n}=\omega n$, with $m=0.2, \omega=7.7$, with $N=1000$ terms in the sum.

FIGURE 6: Graph of $S_{1}(x)$ defined by (69) for the phases $\psi_{n}^{(1)}$ defined by (70 with $m=0.25, \omega=7.7, N=$ 1000.

FIGURE 7 Graph of $S_{2}(x)$ defined by (69) for the phases $\psi_{n}^{(2)}$ defined by (71) with $m=0.5, \omega=8, N=200$.

FIGURE 8: “Golden-mean-log-periodic Weierstrass function" $S_{3}^{(g)}(x)$ defined by (69) with (72) for $m=0.5, \omega=$ $7.7, N=500$.

FIGURE 9: “ $\pi / 4$-log-periodic Weierstrass function" $S_{3}^{(\pi / 4)}$ defined by (69) with (72) and $R=\pi / 4$ for $m=$ $0.5, \omega=7.7, N=500$.

FIGURE 10: "e-log-periodic Weierstrass function" $S_{3}^{(e)}$ defined by (69) with (72) and $R=e=2.718 \ldots$ for $m=0.5, \omega=7.7, N=500$.

FIGURE 11: Singular part $f_{s}(x)$ of the Weierstrass-like function for the regular function $g(x)$ equal to the stretched exponential (82) for $h=5$ (solid line), $h=10$ (dashed line), $h=20$ (dotted line), $h=50$ (dasheddotted line) and $h=100$ (dashed-dot-dotted line), for $m=0.4, \omega=7.7, N=22$.

FIGURE 12: Singular part $f_{s}(x, \delta)$ of the Weierstrass-like function for the regular function $g(x)$ given by (85) for $\delta=-0.1$ (solid line), $\delta=0$ (dashed line) and $\delta=0.1$ (dotted line) for $m=0.4, \omega=7.7, N=27$ ).

FIGURE 13: Weierstrass-type function $f(x)$ for compact $g(x)$ given by (90) with $\nu=2, h=2$ for $m=0.5, \omega=$ $7.7, N=47)$.

FIGURE 14: Evolution of the "singular part" $f_{s}(x)$ corresponding to the compact regular part $g(x)$ (90) for $\nu=2$ with increasing abruptness of $g(x)$ quantified by the exponent $h: h=2$ (solid), $h=5$ (dash), $h=10$ (dot), $h=20$ 
(dash dot), $h=50$ (dash dot dot), $h=100$ (short dash), for $m=0.5, \omega=7.7, N=47$ ).

FIGURE 15: Weierstrass-type function $f(x)$ corresponding to the regular part $g(x)$ defined by (93) with compact support, with $m=0.5, \omega=7.7, N=47$.

FIGURE 16: Weierstrass-type function $f(x)$ corresponding to the regular part $g(x)$ defined by (96) with compact support, with $m=0.5, \omega=7.7, N=47$. 
Fig. 1

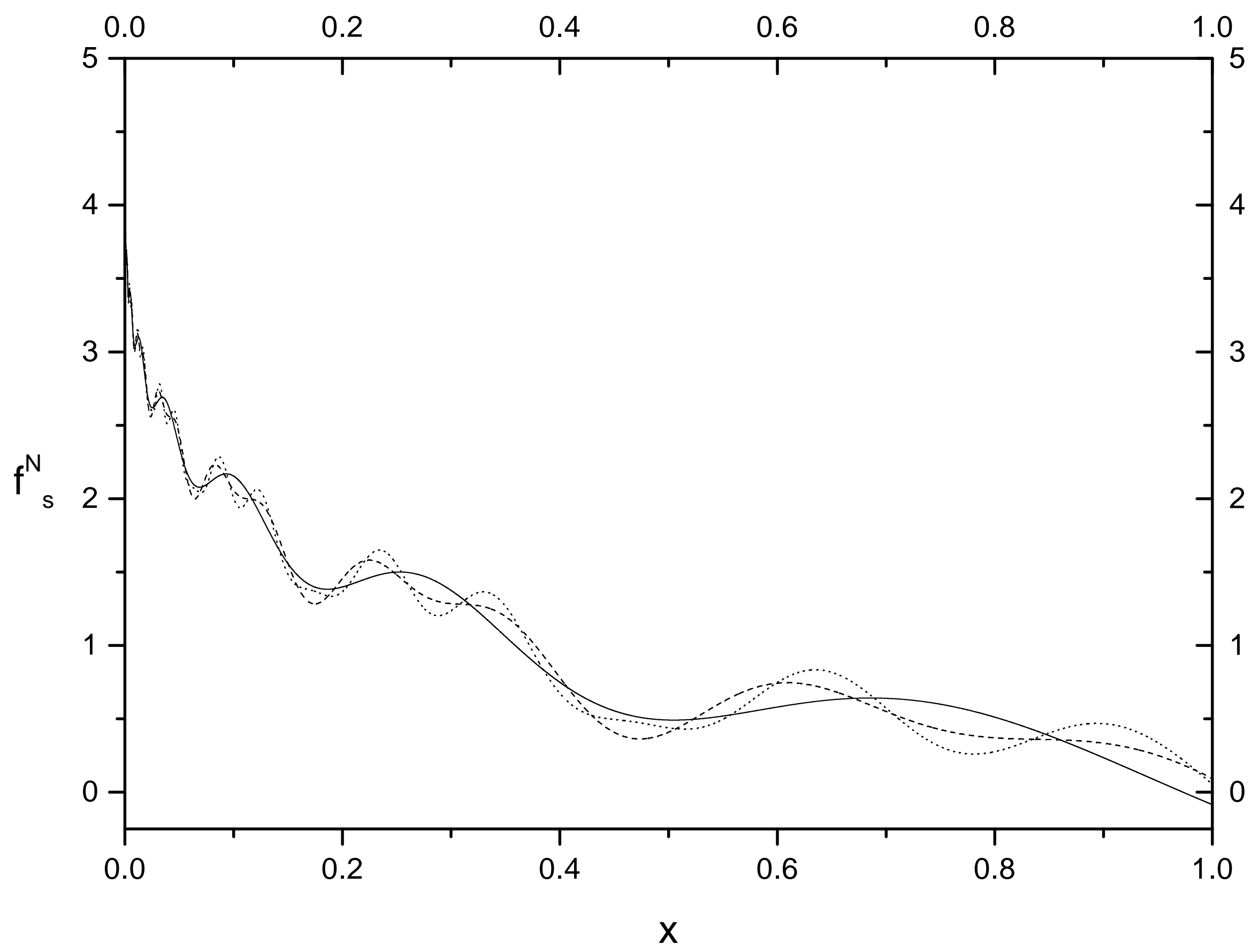


Fig. 2 (a)

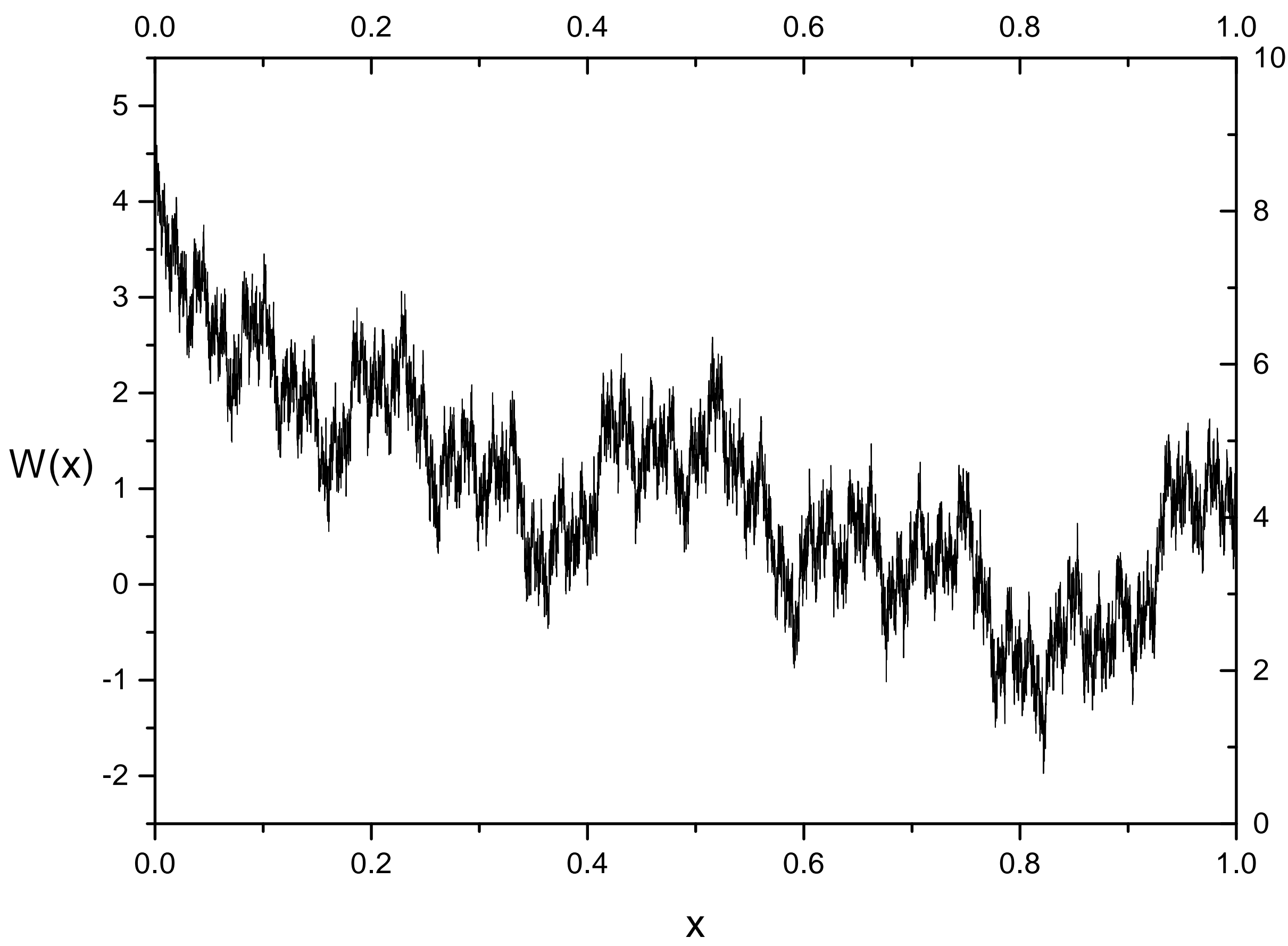


Fig. 2 (b)

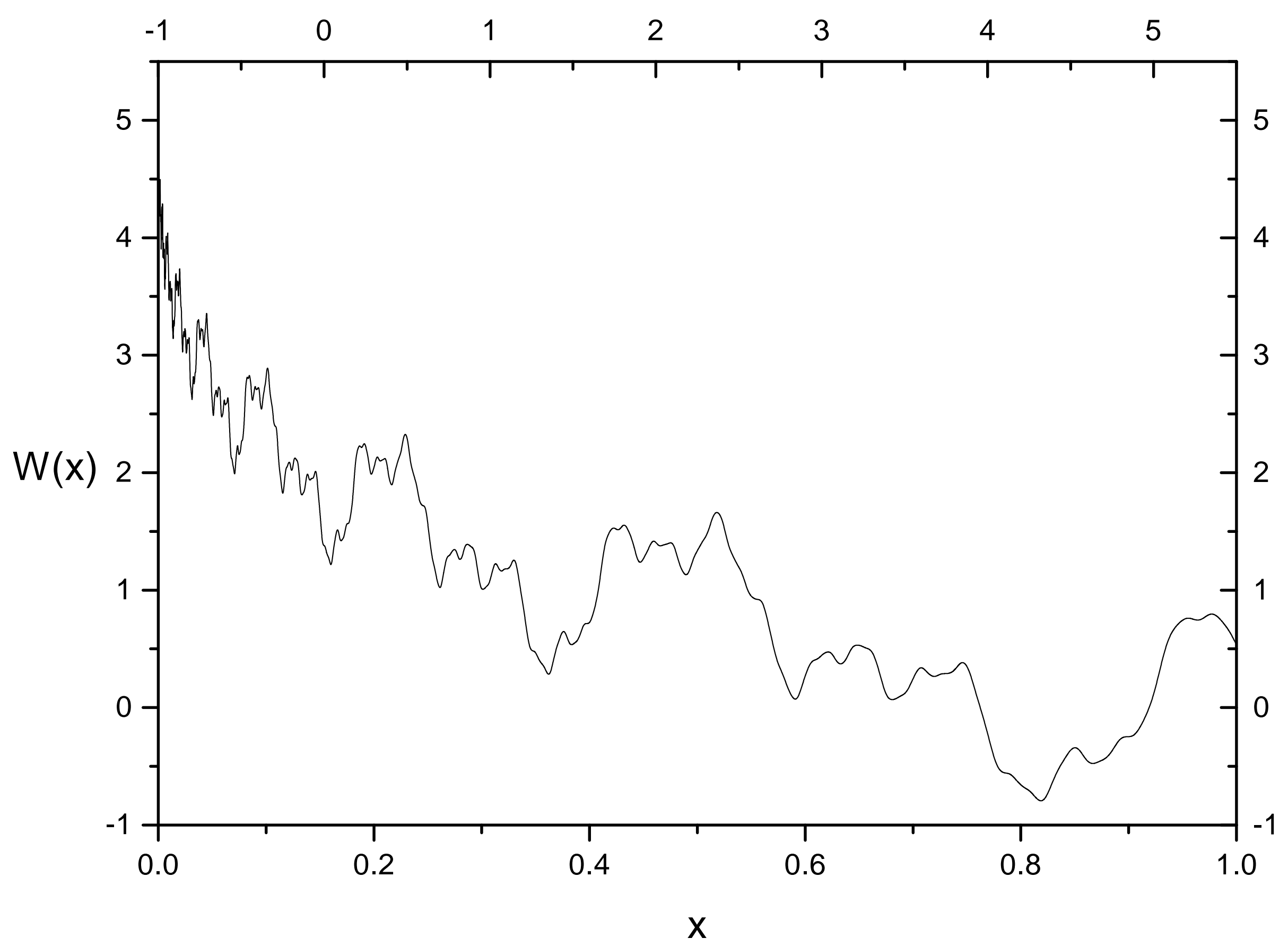


Fig. 2 (c)

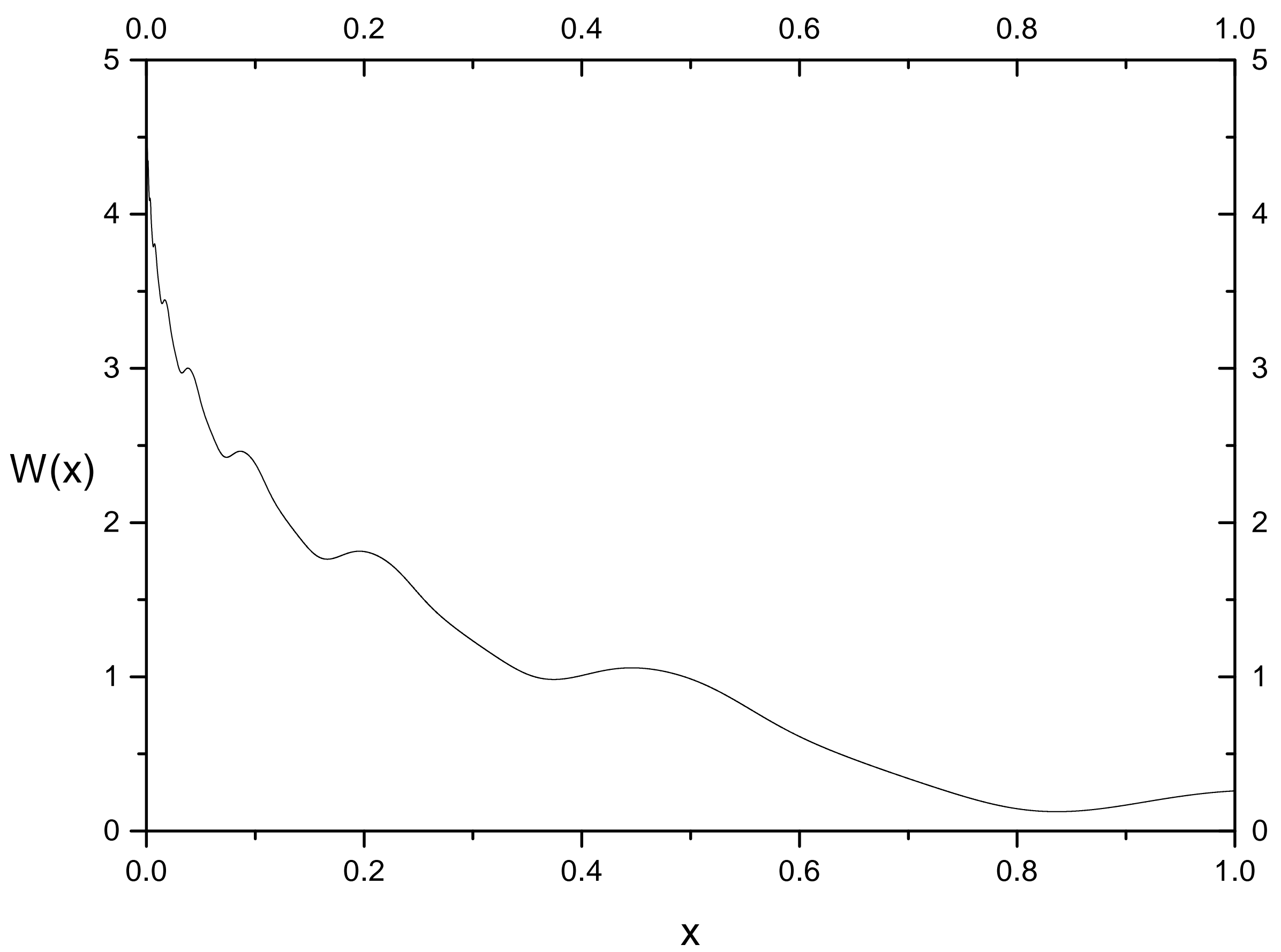


Fig. 2 (d)

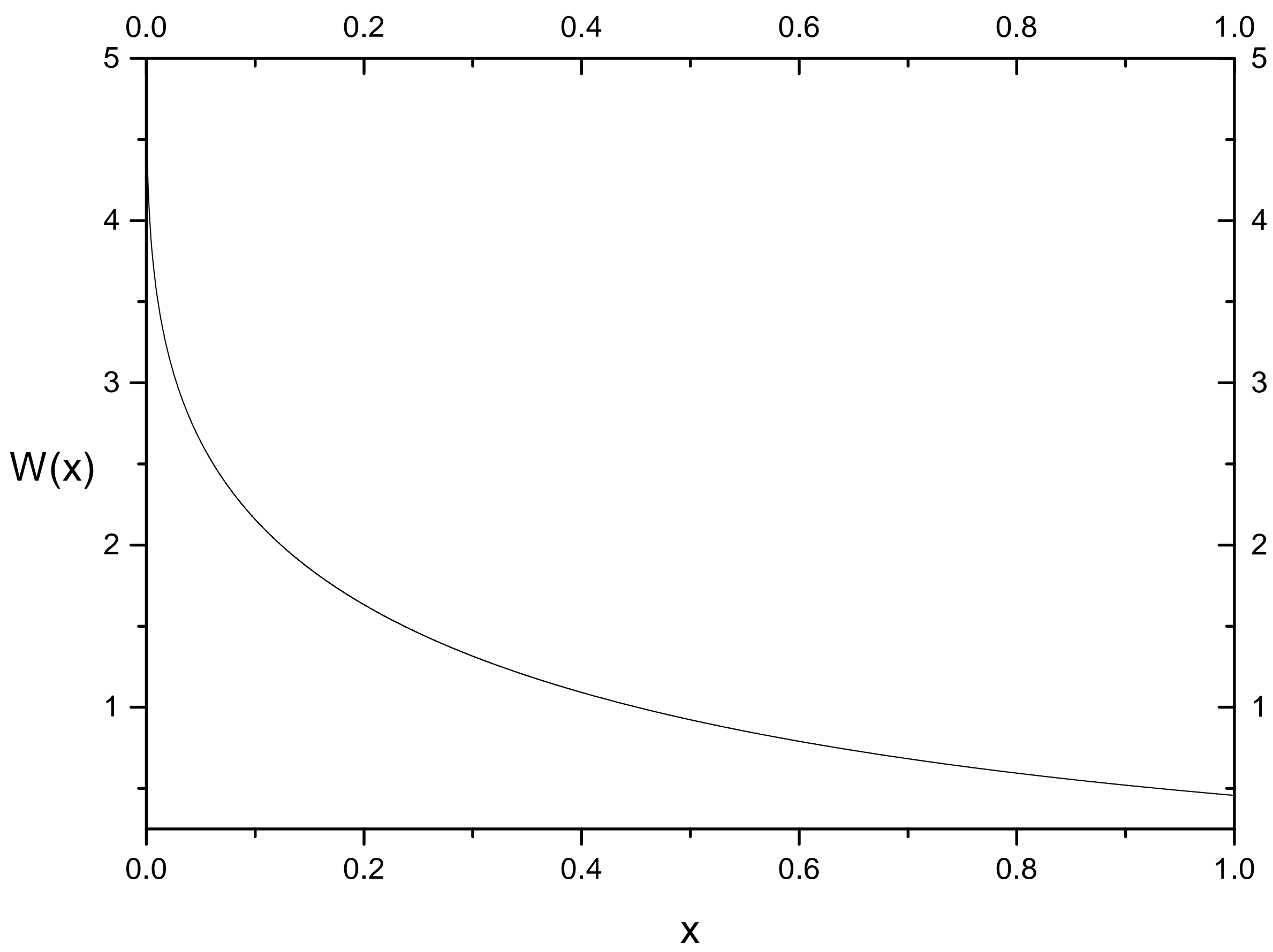


Fig. 3 (a)

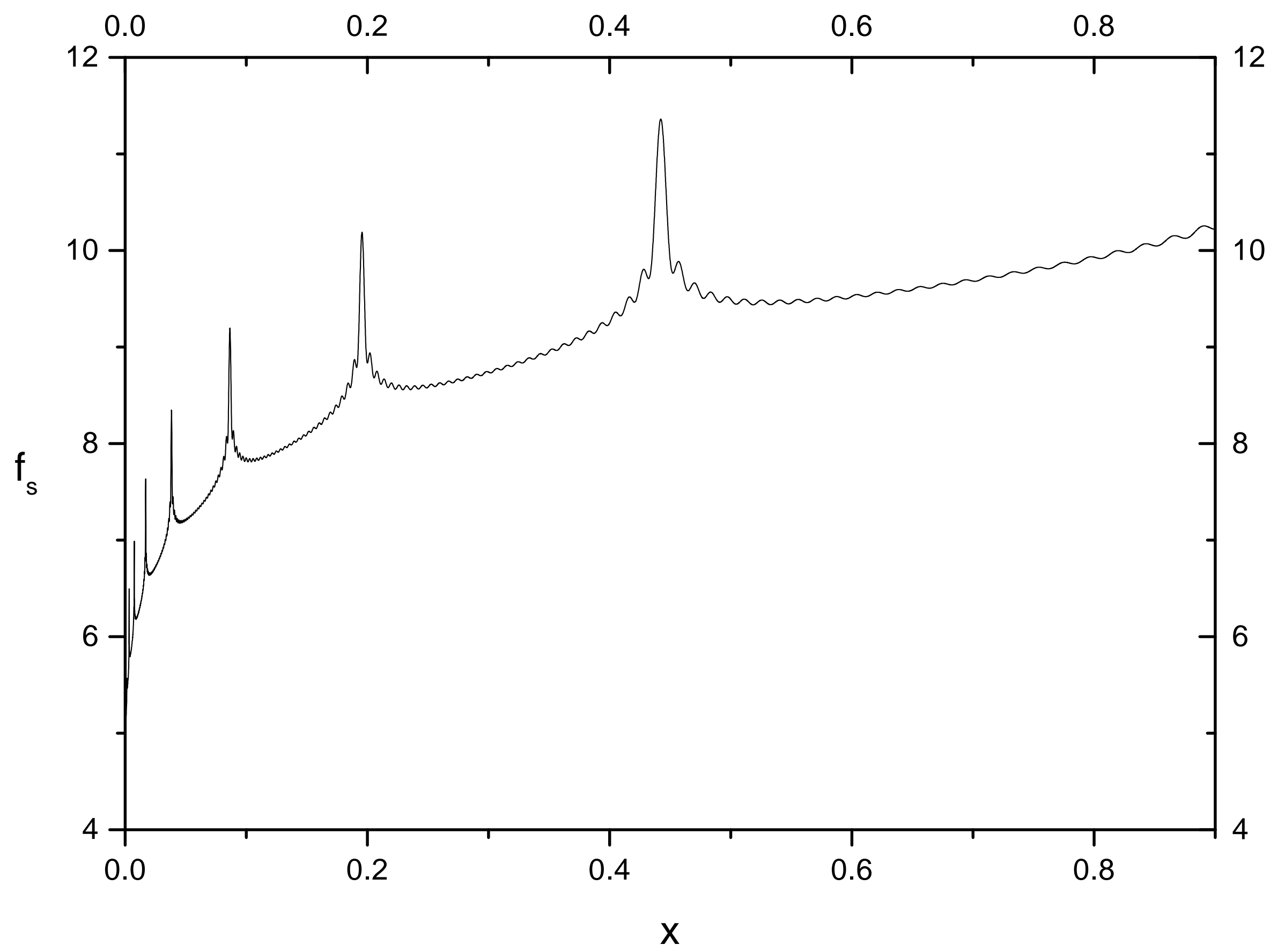


Fig. 3 (b)

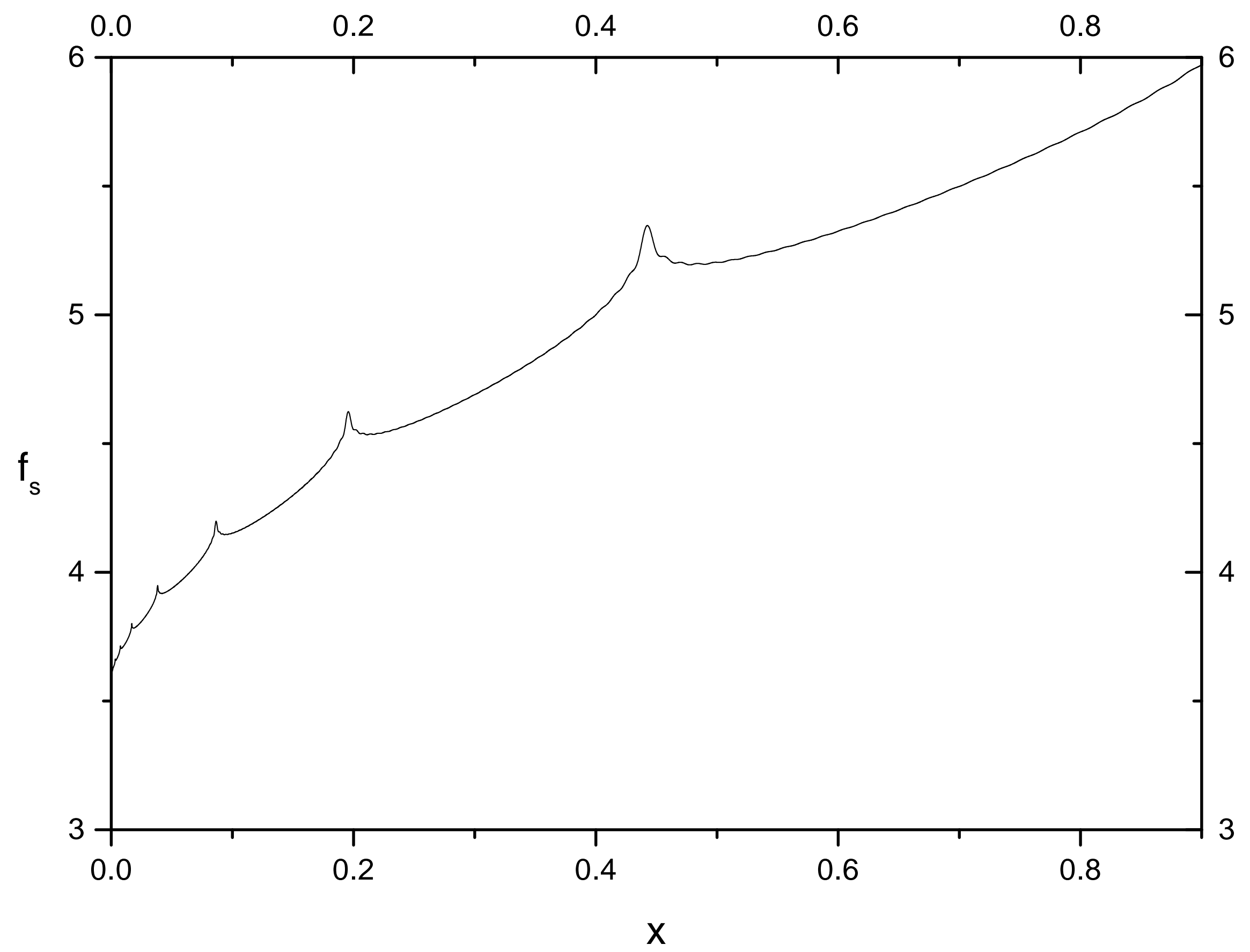


Fig. 4

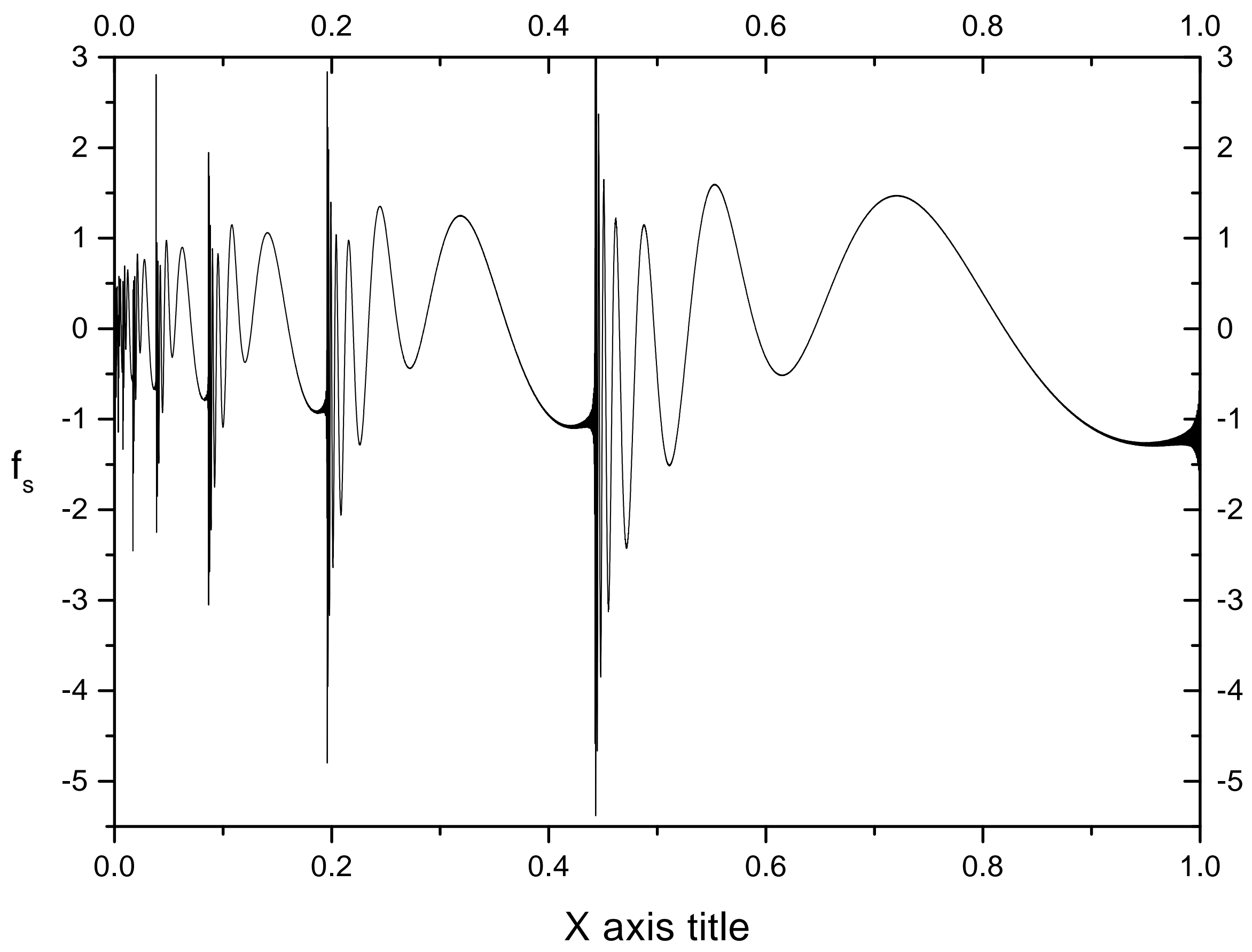


Fig. 5

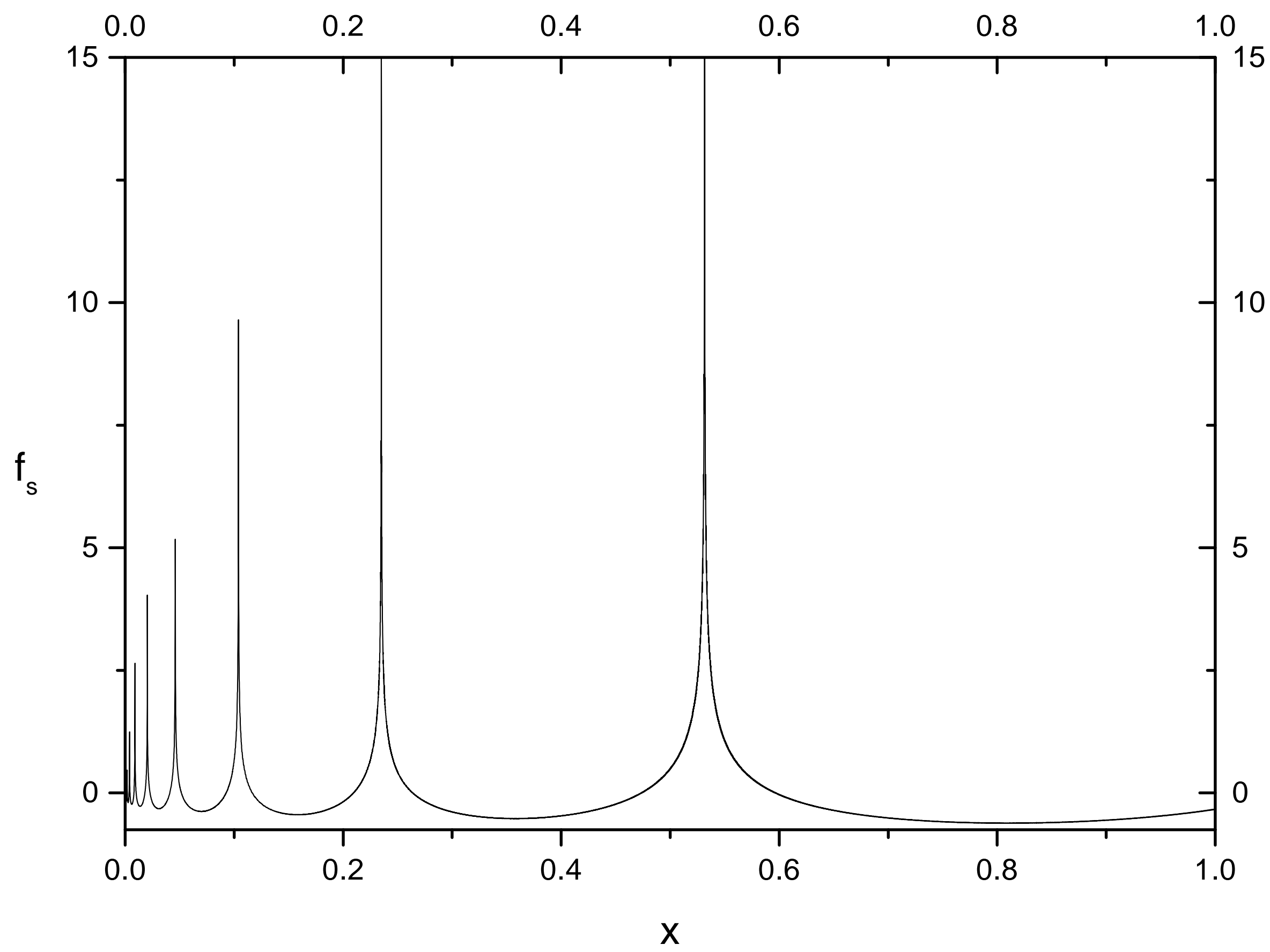


Fig. 6

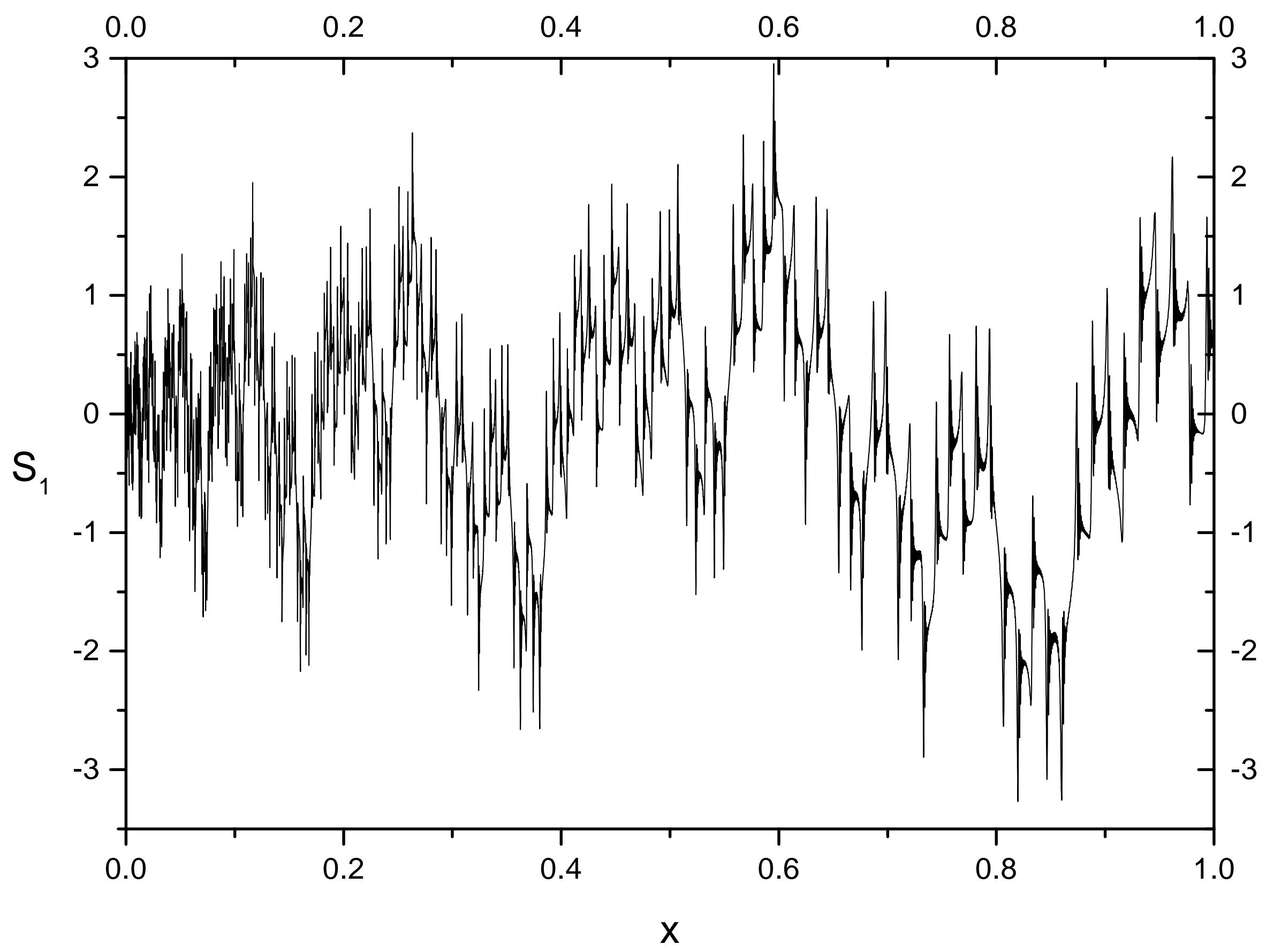


Fig. 7

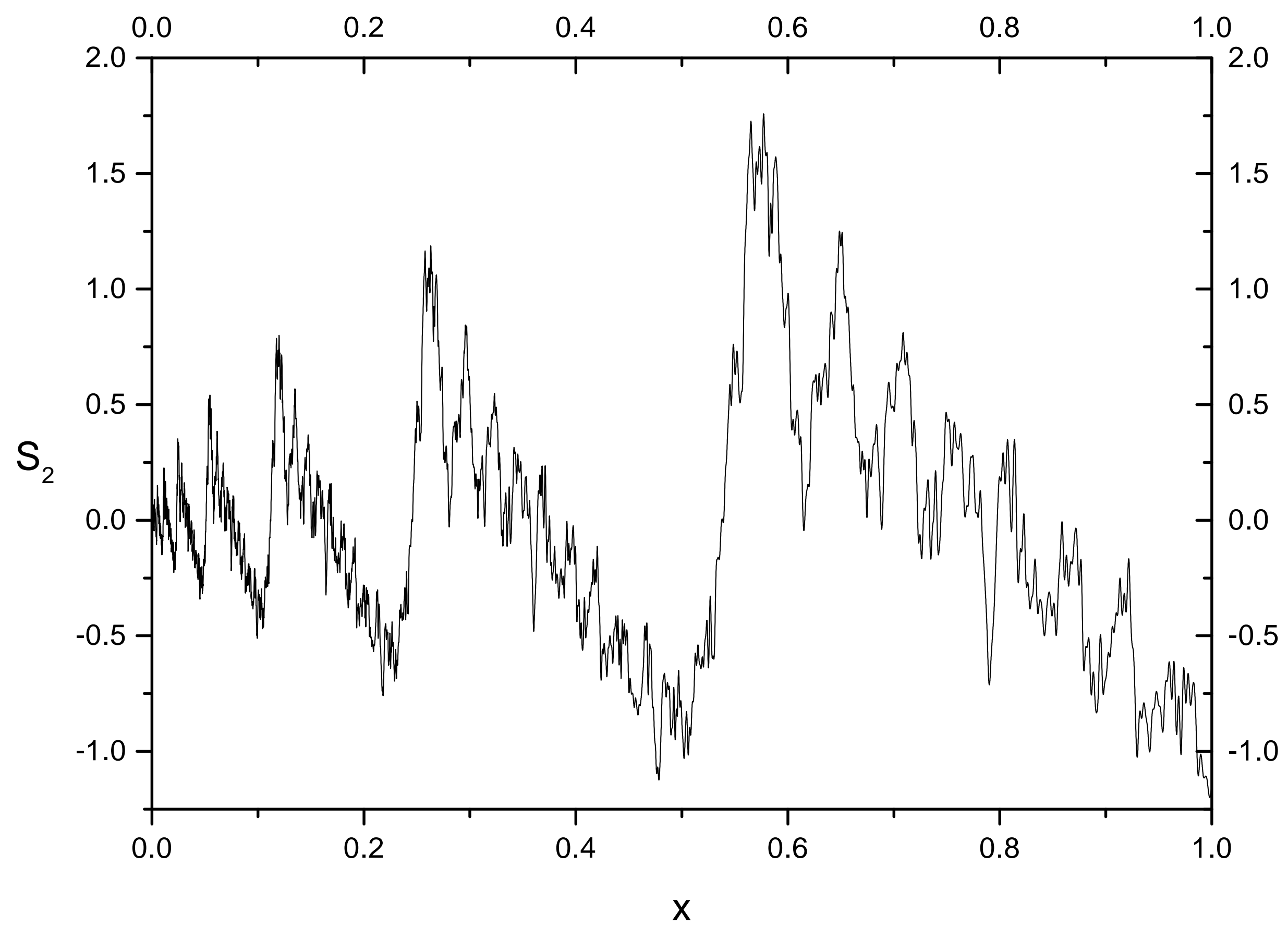


Fig. 8

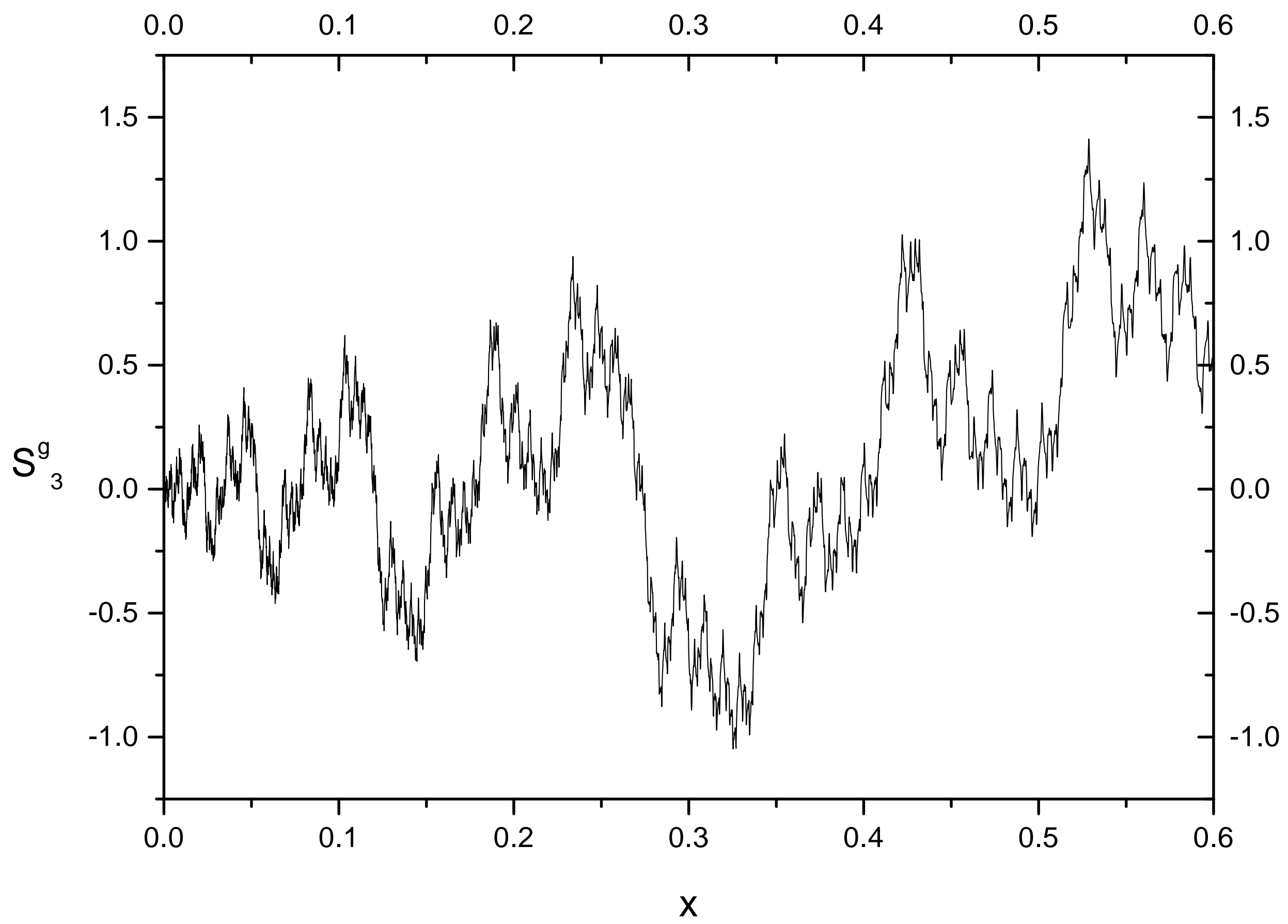


Fig. 9

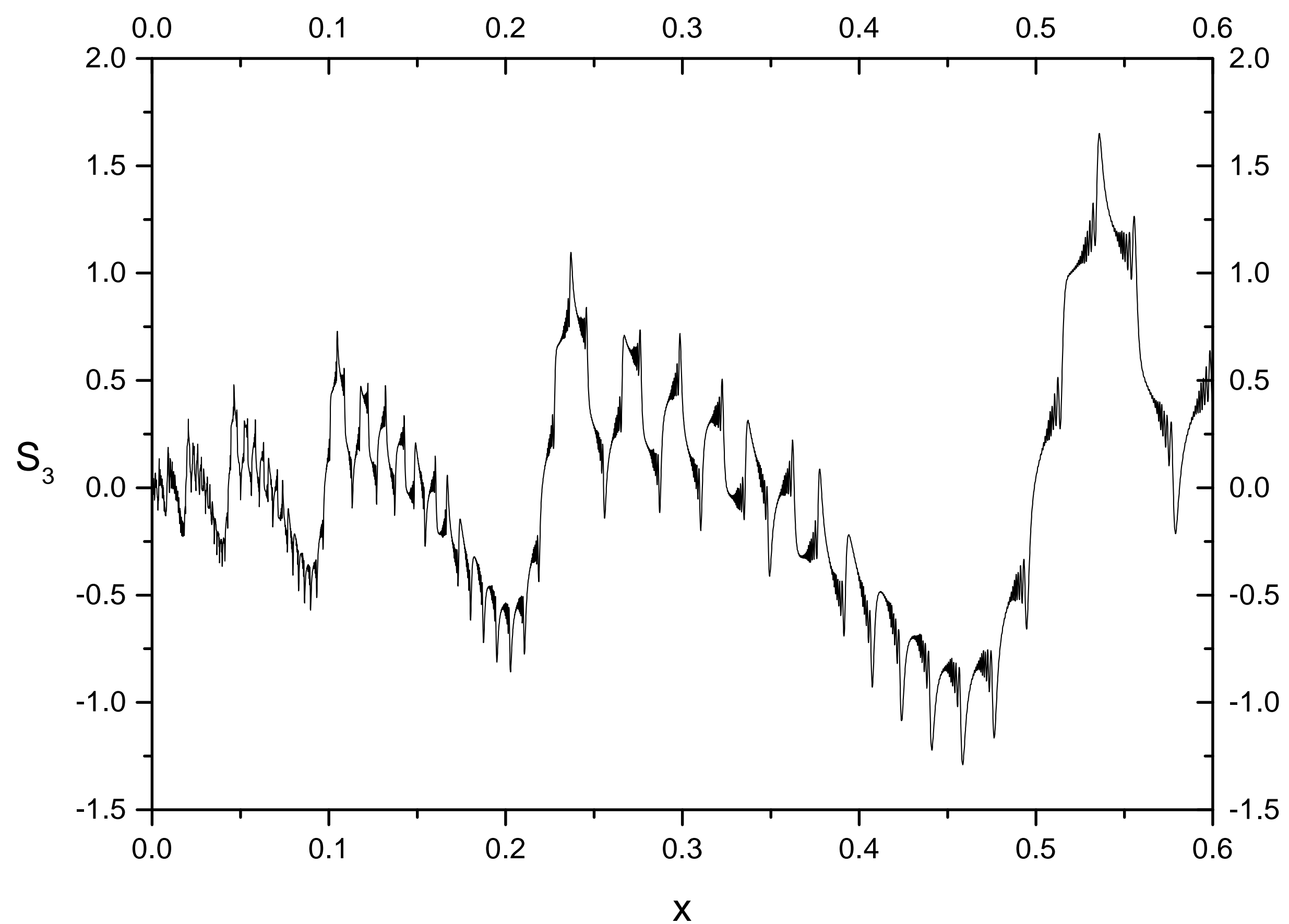


Fig. 10

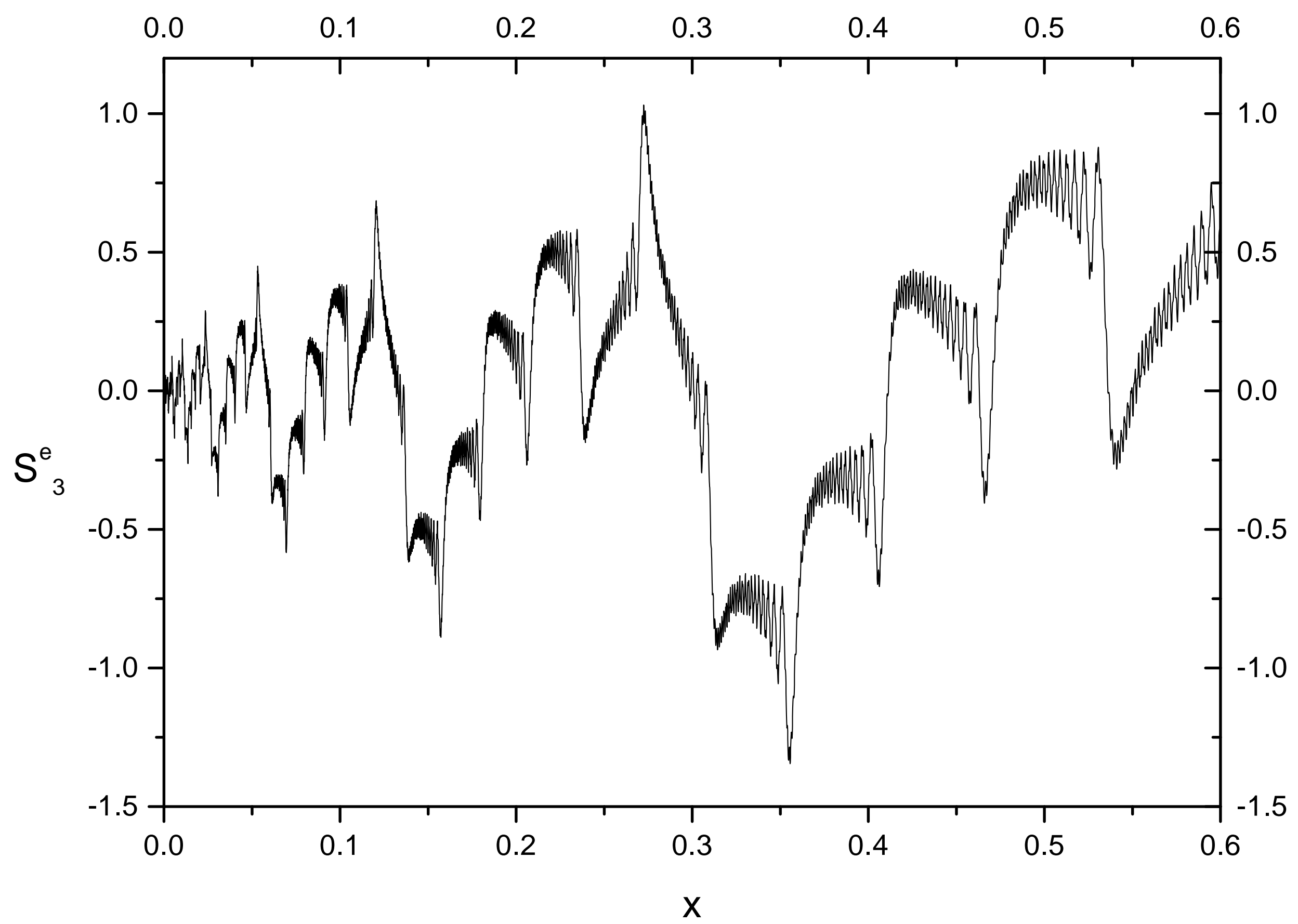


Fig. 11

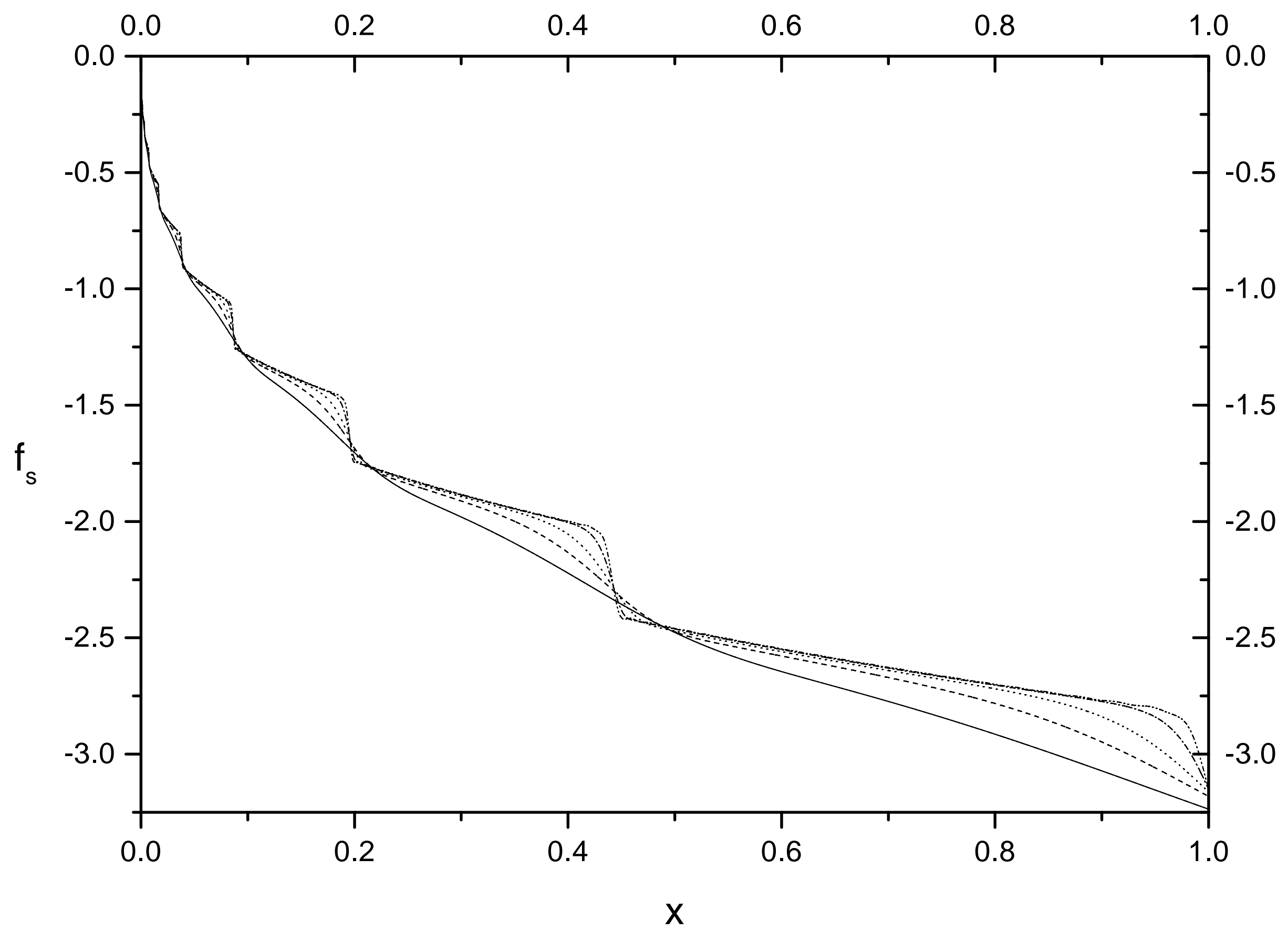


Fig. 12

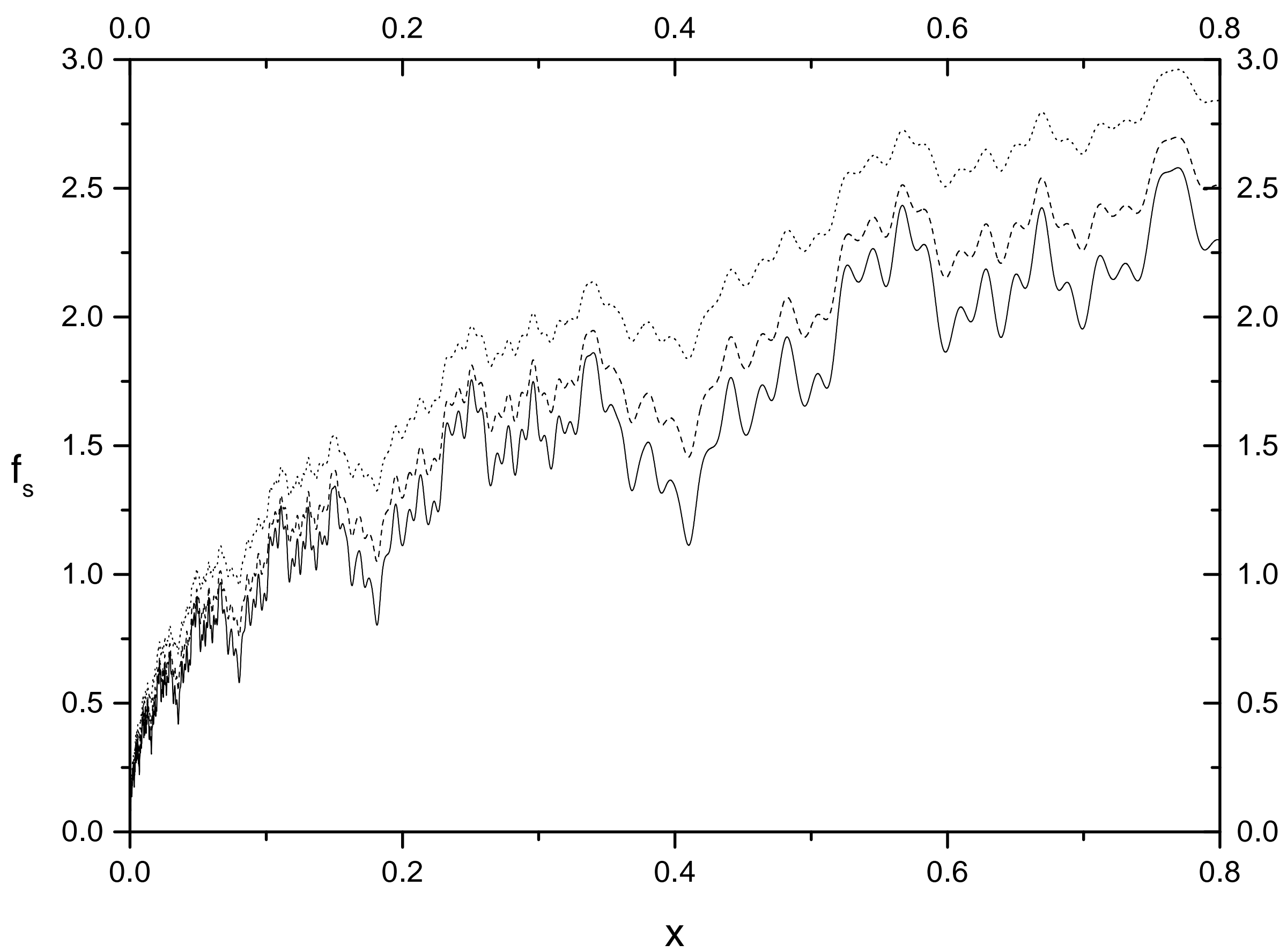


Fig. 13

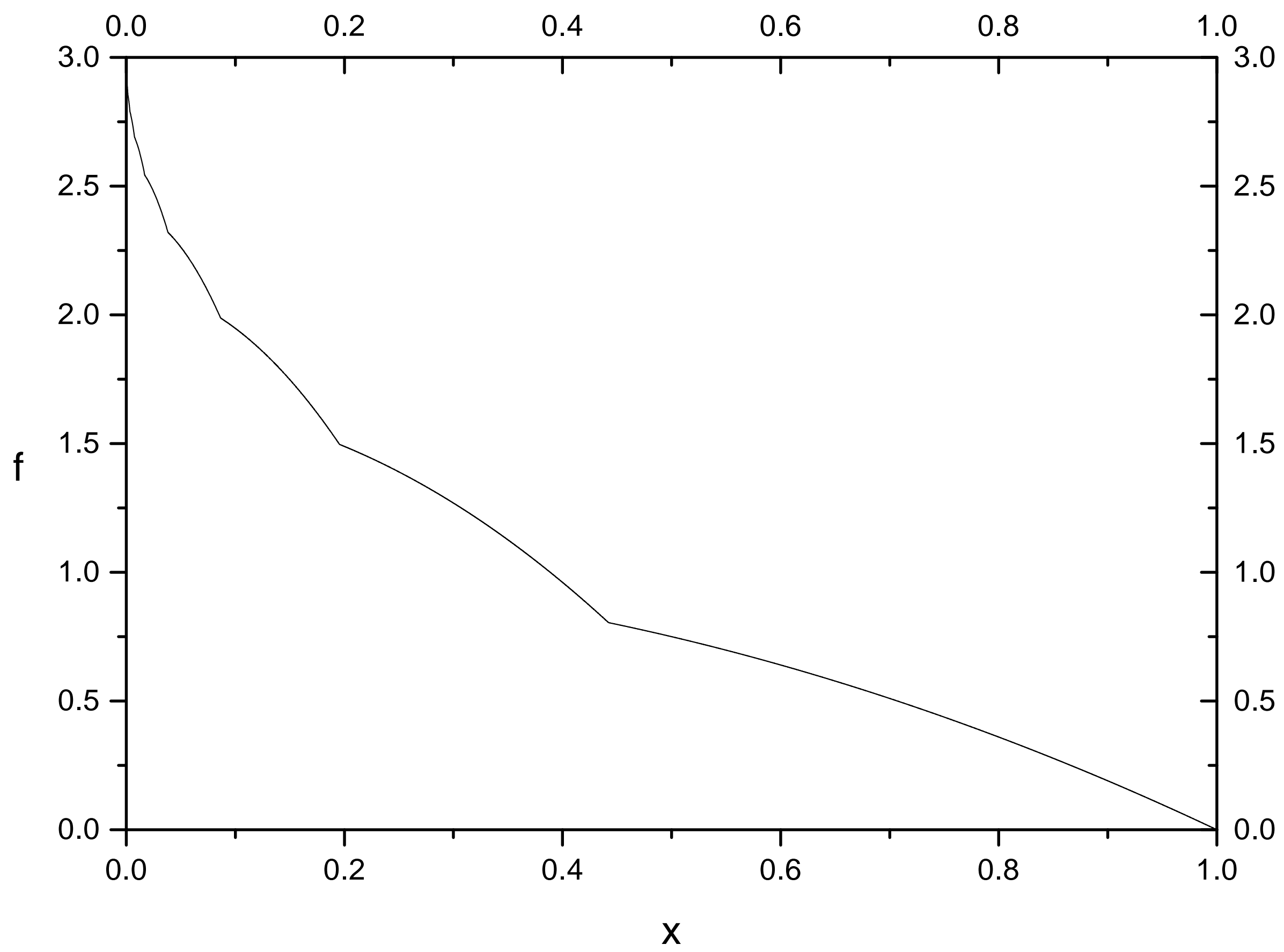


Fig. 14

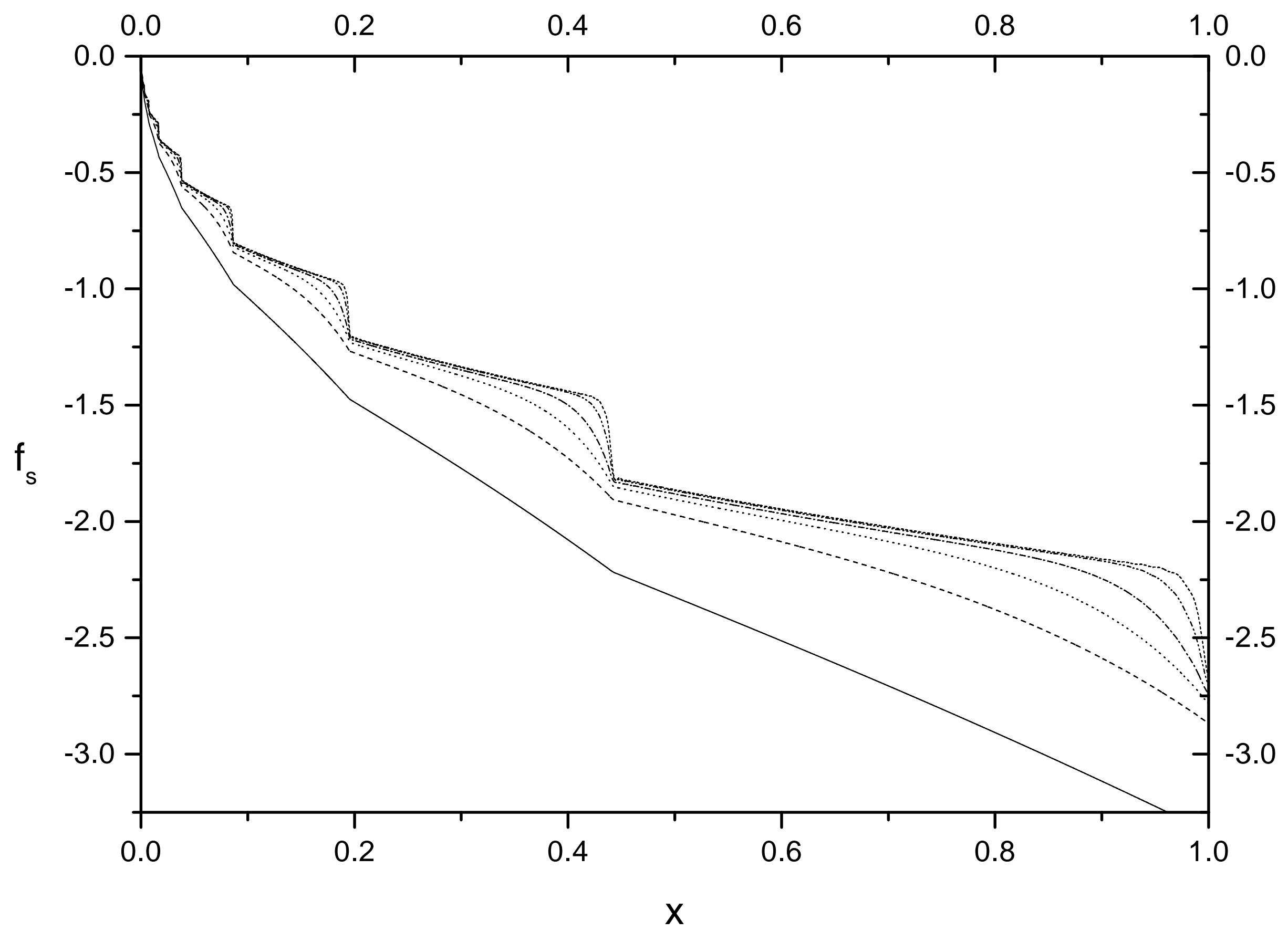


Fig. 15

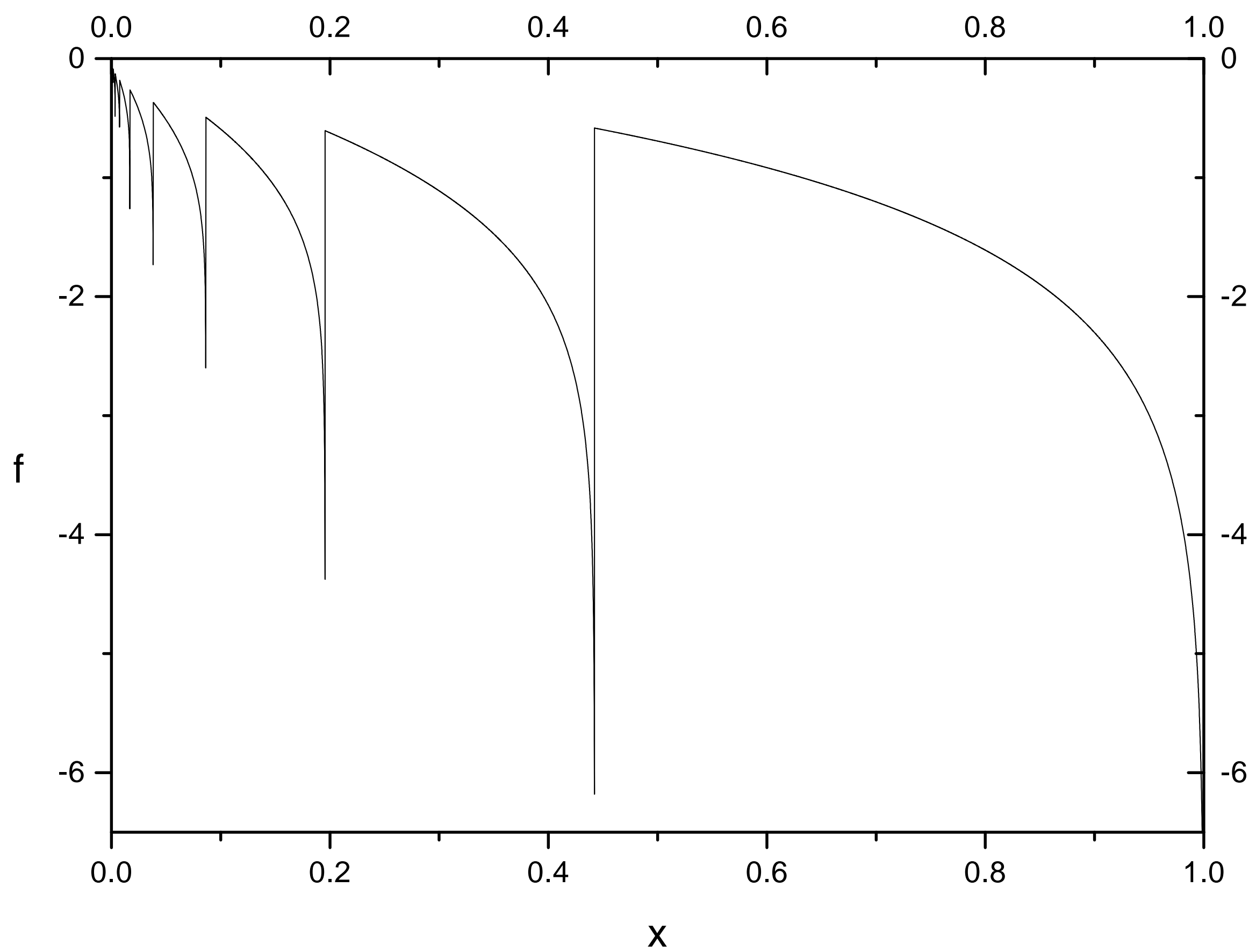


Fig. 16

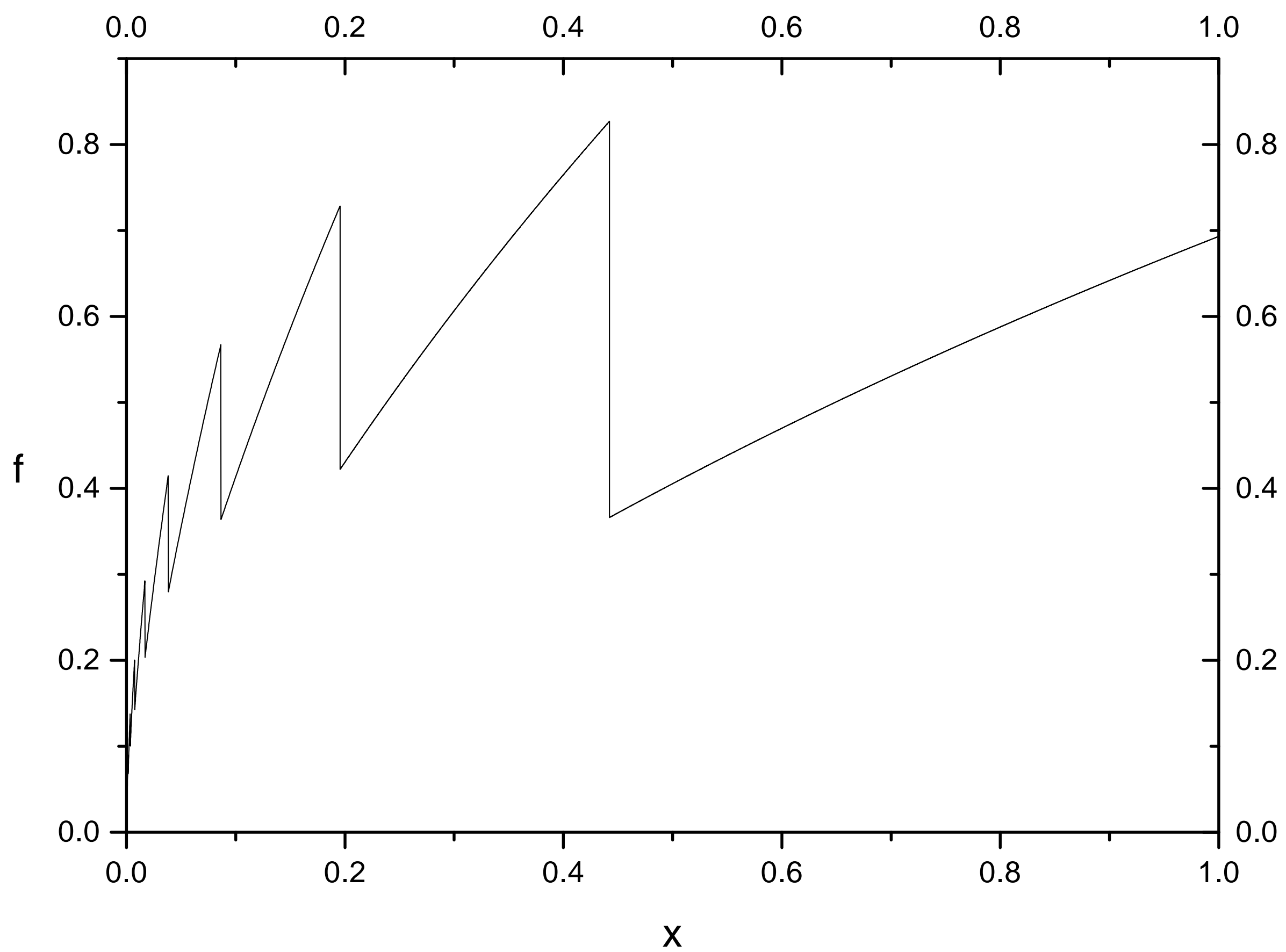

